\title{
Model of Universe as Described by Dynamic Universe Model
}

\author{
Satyavarapu Naga Parameswara Gupta \\ Bhilai Steel Plant, Bhilai, India \\ Email:snp.gupta@gmail.com,snp.gupta@hotmail.com
}

How to cite this paper: Gupta, S.N.P. (2019) Model of Universe as Described by Dynamic Universe Model. Open Journal of Modelling and Simulation, 7, 41-78. https://doi.org/10.4236/ojmsi.2019.71003

Received: July 30, 2018

Accepted: November 24, 2018

Published: November 27, 2018

Copyright $\odot 2019$ by author and Scientific Research Publishing Inc. This work is licensed under the Creative Commons Attribution International License (CC BY 4.0).

http://creativecommons.org/licenses/by/4.0/

\begin{abstract}
In this paper we will see the model of Universe according to Dynamic Universe Model of Cosmology by visualizing various processes that are happening in the Universe as per experimental evidences. For simplifying the matter here, we will see in part 1: about the Galaxy life cycle, where the birth and death of Galaxies discussed. Probably Universe gives guidance for the movement of Galaxies. We call this Part 1: Thinking and Reproducing Universe or Mindless Universe? (Galaxy life cycle). We see every day Sun, Stars, Galaxies etc., dissipating enormous energy in the form of radiation by the way of fusion of Hydrogen to helium. So after sometime all the Hydrogen is spent and Universe will die, is it not? ... Dynamic Universe Model says that the energy in the form of electromagnetic radiation passing grazingly near any gravitating mass changes in frequency and finally will convert into neutrinos (mass). Hence Dynamic Universe Model proposes another process where energy will be converted back into matter and the cycle energy to mass to energy continues, sustaining the Universe to maintain this present status for ever in this form something like a Steady state model without any expansion. This we will see in Part 2: Energy - Mass - Energy Cycle. After converting energy into mass "how various elements are formed and where they are formed?" will be next logical question. Dynamic Universe Model says that these various particles change into higher massive particles or may get bombarded into stars or planets and various elements are formed. Here we bifurcate the formation of elements into 6 processes. They are for Elementary particles and elements generated in frequency changing process, By Cosmic rays, By Small stars, By Large Stars, By Super Novae and Manmade elements By Neutron Stars. This we will discuss in Part 3: Nucleosynthesis.
\end{abstract}

\section{Keywords}

Dynamic Universe Model, Hubble Space Telescope (HST), SITA Simulations (SITA-Simulation of Inter Intra Tautness Attraction Forces Used by Dynamic 
Universe Model), Singularity-Free Cosmology, Blue Shifted Galaxies, Red Shifted Galaxies, Grazing Radiation Frequency Changes, Formation of Elements, Nucleosynthesis Dynamic Universe Model, Energy to Mass Conversion, Methods: N-Body Simulations-Gravitation-Cosmology

\section{Introduction}

Energy is produced by stars. Matter consists of many elements and compounds. Both of these are maintaining balance and sustenance. There is energy, there is matter and there are Galaxies and Stars in the universe. None of these quantities ever goes up or down, and a balance is always maintained. According to Dynamic Universe Model none of the above dominates the Universe. No energy dominated era, No matter Dominated era, always there is balance; and the balance is maintained dynamically ever. The Universe stays like this for ever. Some Galaxies may quench, new Galaxies may bourn but the Universe as a whole will stay in this condition without any birth or death. With these concepts the author makes a small scientific attempt to explain how all these processes will be possible within the per-view of Vak's Dynamic Universe model of Cosmology.

This is a consolidating paper. This paper is based on three main papers that are published by the author recently and many other papers published by many others scientists and astrophysicists. And as a matter of fact, ninety nine percent of the concepts in this paper are well published already by many authors in the world including me. See the published papers at references [1] [2].

In this paper the author tries to cover some of the main processes of the Universe and visualize the "Dynamic Universe Model" as a Universe model. What are these processes? Three main processes are identified in this paper. Three parts of this paper describe these main processes.

The Galaxies in the Universe took birth at different times. Many papers were published telling that the Galaxies have ages as long as 46 billion years to as low as 10 billion years. If Bigbang is having age of 13.8 billion years, why so many different ages? Some Galaxies have very high age and some have lower age? This process is covered in "Part 1: Thinking and Reproducing Universe or Mindless Universe? (Galaxy life cycle) ...”. The main principle in this part 1 is whatever takes birth in the Universe will die sooner or later. See the paper "Distances, Locations, Ages and Reproduction of Galaxies in our Dynamic Universe" in this part.

We see all the stars burning fuel and radiating energy, and slowly they die out and go to the outer rims of Galaxies. New stars take birth in star forming clouds of the Galaxies. Who will give the matter to these areas for formation of new stars? What is the process? Will all the energy produced by the stars spread out go into nowhere, or to some outer space? Dynamic Universe Model proposes a CLOSED universe model that no energy goes out, and covering these areas and proposes another process, a missing link "How the energy is transformed back to matter in "Part 2: Energy - Mass - Energy Cycle". 
The matter so condensed from energy will be in the form of some nucleons like neutrinos, electrons or positrons. From these various elements are formed and various compounds are formed. You will find the discussion on these processes which happen mostly inside stars and in the core of the planets. These processes are dealt in Part 3: Nucleosynthesis and Bio-Intelligence origins.

The author published three papers covering each part, describes the process which is leading in that part. This paper provides the links between all these well published material through this Vak's Dynamic Universe Model of Cosmology. This paper will try to cover all the gray areas and missing links in the published material and papers.

This paper is the basis for Eighth book in the series on Dynamic Universe Model: SITA, (SITA-Simulation of Inter intra Tautness Attraction forces) which is being written.

Newtonian two-body problem used differential equations. Einstein's general relativity used tensors [3], which in turn unwrap into differential equations. Dynamic Universe Model uses tensors that give simple equations with interdependencies. Differential equations will not give unique solutions. Whereas Dynamic Universe Model gives a unique solution of positions, velocities and accelerations; for each point mass in the system for every instant of time. This new method of Mathematics in Dynamic Universe Model is different from all earlier methods of solving general N-body problem.

This universe exists now in the present state, it existed earlier, and it will continue to exist in future also in a similar way. All physical laws will work at any time and at any place. Evidences for the three dimensional rotations or the dynamism of the universe can be seen in the streaming motions of local group and local cluster. Here in this dynamic universe, both the red shifted and blue shifted Galaxies co-exist simultaneously.

A point to be noted here is that the Dynamic Universe Model never reduces to General relativity on any condition. It uses a different type of mathematics based on Newtonian physics. This mathematics used here is simple and straightforward. As there are no differential equations present in Dynamic Universe Model, the set of equations give single solution in $\mathrm{x} y \mathrm{z}$ Cartesian coordinates for every point mass for every time step. All the mathematics and the Excel based software details are explained in the three books published by the author [4] [5] [6] In the first book, the solution to N-body problem-called Dynamic Universe Model (SITA) is presented; which is singularity-free, inter-body collision free and dynamically stable. This is the Basic Theory of Dynamic Universe Model published in 2010 14. The second book in the series describes the SITA software in EXCEL emphasizing the singularity free portions. It explains more than 21,000 different equations (2011). The third book describes the SITA software in EXCEL in the accompanying CD/DVD emphasizing mainly HANDS ON usage of a simplified version in an easy way. The third book contains explanation for 3000 equations instead of earlier 21000 (2011) [6]. 
SITA solution can be used in many places like presently unsolved applications like Pioneer anomaly at the Solar system level, Missing mass due to Star circular velocities and Galaxy disk formation at Galaxy level etc. Here we are using it for prediction of blue shifted Galaxies.

Dynamic Universe model does NOT depend on speculation. It is based on hard observed facts. I am writing these sentences especially as cosmology is becoming more and more speculative today.

\section{Part 1: Thinking and Reproducing Universe or Mindless Universe? (Galaxy Life Cycle)}

\subsection{Galaxy Life}

Sir Jagadish Chandra Bose [7] clearly demonstrated that the Trees, stones etc also behave like other life forms and react to alkalis, acids and poisons like arsenic. The Universe also must have life or some form of intelligence of its own. To demonstrate this, the author wants to show two abilities, the first one is present in any life form called "Reproduction", which the Galaxies also possesses. The assumption here is that the Galaxies will take birth and die (quench) and are off springs of the Universe. The concepts of Dynamic Universe model are used for exhibiting this property.

Another property is the Galaxies will get continuous guidance for their movement in the Universe. They move according to the vectorial force called Universal Gravitational Force (UGF) acting on that Galaxy. This UGF is the resultant of the vector forces acting that Galaxy by all the other masses of the Universe at that instant of time. So with this property of the Universe the said Galaxy will move and the Universe give continuous guidance to move the Galaxy accordingly.

Bigbang cosmologists say that the Universe has been around for 13.8 billion years, but we observe through various telescopes like HST (Hubble Space Telescope) etc, we can see for 46 billion light-years. How is this possible?

According to Dynamic Universe Model, the Universe as a whole there is no beginning or end. Galaxies will be taking birth and quench. They are all different ages. Not bourn simultaneously. It is not necessary that the whole Universe was begin once some long time ago. We will find Galaxies of different ages, some have as large age like 46 billion years, and some have an age as less as 10 billion years. Bigbang has an age of 13.7 billion years the mainstream says. Why Bigbang age has so much difference with observations with Galaxy ages?

There are many distant Galaxies whose distance is about 30 Giga light years. There are Galaxies which were born just after 400 million years after Bigbang and which were born 6 to 7 billion years after Bigbang. In Dynamic Universe Model all the Galaxies are distributed at different distances and the Universe looks similar as it is now. On 01March2016 the news of the discovery about the observation of most distant Galaxy "GNZ11 or GN11" having an light travel distance 13.4 Giga light years \& co-moving distance of 32 Giga light years and 
having a red shift $\mathrm{z}$ of 11.1 created quite a stir. There are many such Galaxies like EGSY8p7 (with $z=8.68$, age $=13.2$ ) and EGS-zs8-1 (with $z=7.73$, age $=13.04$ ). To show that Universe exists further to GNz11, a Galaxy at distance of 100 times 13.4 Billion light years $(1.26862 \mathrm{E}+28$ meters) is simulated and named it as GNz11 in this simulation. 132 more galaxies were assumed in the range $(3.02001 \mathrm{E}+26$ to $1.26862 \mathrm{E}+28)$ meter. Later distance of the first Galaxy was reduced by $50 \%$ and found the graphs of Universe become similar in both the simulations after 102 iterations.

The stars in a Galaxy emit light and other electromagnetic radiation. Slowly life of the stars and hence subsequently the life of Galaxy will come to end. Galaxies tend to evolve from spiral to elliptical structure and they perish to form Blue clouds known as Galaxy "quenching" (Death of Galaxy). Hence we can say that our Universe had reproduction ability, which is a very slow process. Universe produces new Galaxies, and the already formed Galaxies perish slowly. Ours is single universe and is a closed one. In other words, our Universe reproduces its Galaxies, as and when light and other electromagnetic radiation condenses to form enough matter.

In Dynamic Universe Model, like in Steady State model, the Universe looks similar as it is now. There is no creation of mater in empty space. The electro-magnetic radiation shifts its frequency near huge gravitating masses. In this model, different Galaxies born and perish according to their own age and life cycle. They will have different birth dates and ages. In other words, they won't have exactly same birth-date for every candidate. This is one of the starting and basic assumptions or axiom of Dynamic Universe Model. There are many galaxies whose age \& light travel distance are near about 13 billion years, that is about 0.5 billion years after Bigbang. Bigbang says first stars took birth after 3 to 4 Billion years, Galaxy formation took more time. All these started from the initial Gas after Bigbang. Most recently found is GNz-11, it was found $1^{\text {st }}$ March 2016. Details of four of the most distant Galaxies are given below. The positions of these Galaxies are distributed in different directions over the sky.

\subsubsection{GN-z11}

P. A. Oesch, G. Brammer, [8] and others are the first people discovered and submitted the paper on 1 Mar 2016 about this GNz-11. GN-z11 has a spectroscopic redshift of $\mathrm{z}=11.1$. This is in the constellation Ursa Major, It existed 13.4 billion years ago, just 400 million years after the Big Bang; and is the oldest Galaxy with a comoving dist $=32$ billion ly.

\subsubsection{EGSY8p7 (EGSY-2008532660)}

next distant galaxy, with a spectroscopic redshift of $z=8.68$ (photometric redshift 8.57), a light travel distance of 13.2 billion light-years from Earth, an age of 13.2 billion years, detected by Adi Zitrin, Ivo Labbe et al July 2015 [9].

\subsubsection{EGS-zs8-1}

Is in the constellation of Boötes and is first spotted by Yale astronomer Pascal 
Oesch [10] in Hubble Space Telescope images in 2013. It has a redshift $z=7.73$, corresponding to a light travel distance and age of about 13.04 billion light years from Earth. The galaxy has a comoving distance of about 30 billion light years from Earth.

\subsubsection{UDFy-38135539}

(Also known as "HUDF.YD3"): is in the Hubble Ultra Deep Field (UDF) identifier for a galaxy which was calculated as of October 2010 to have a light travel time of 13.1 billion years with a present proper distance of around 30 billion light-years.

\subsection{Age of Universe}

The concepts by Lemaitre, Edwin Hubble and Alexander Friedmann in the 1920s became known as the Big Bang theory, gave the universe some age. Then at that time Hubble constant approximated as $550 \mathrm{~km} / \mathrm{s} / \mathrm{Mpc}$, the universe had an age as 1.8 billion years which is less than then age of Earth as 2 billion years. The errors by Walter Baade in 1952, and Allan Sandage et al, in 1958 forced a correction in the Hubble constant, to $75 \mathrm{~km} / \mathrm{s} / \mathrm{Mpc}$. This gave an age as $13.0 \mathrm{bil}-$ lion years, which is much higher than the re-measured age of the Earth of 4.55 billion years. Later the Hubble constant found by Sandage and Gustav Tammann in 1970s gave values around $50-60 \mathrm{~km} / \mathrm{s} / \mathrm{Mpc}$, give an age of $16-20$ billion years, consistent with globular cluster ages. Through Hubble space telescope (HST) got a value of $72 \mathrm{~km} / \mathrm{s} / \mathrm{Mpc}+$ or $-10 \%$. WMAP and Planck spacecraft gave an age of the universe at $13.80+$ or $-0.3 \%$ billion years. Thus this cosmic age problem was discussed in the last 120 years in various ways and the outcomes of discussions were different in every 20 years. It is most probably Special relativity not applicable or redshift is not a measure of velocity of Galaxy, as distant galaxies are moving away from us at much higher velocities than speed of light. BUT according to Dynamic Universe Model there is no Birth or Death of universe, it will be continuing in this state. Only individual Galaxies, Stars etc., will have birth and death.

\subsection{Ages of Galaxies}

The Galaxy star-formation is highest when ages of Many Galaxies is about 10 billion years, even though it was found that many massive galaxies had age higher than 13.5 billion years (comoving distance of 32 billion light years) or even may be more than 90 billion years. See these three references [11] [12] $[13] \ldots$

On the other hand many Galaxies were found having lesser age than 8 billion years. We all know that the Bigbang age is about 13.8 billion light years. Traditionally the hierarchical models or "tau models" (for a General explanation see [14]) were used by the astronomers for the last 40 years or so based on probably incorrect assumptions "the star-formation starts off high and drops off exponentially" and "a galaxy starts with a fixed reservoir of gas and its star formation 
must keep dropping as that gas is used up". Astronomers found the drawback of "tau models" as these models "globally-averaged cosmic star-formation rate" took place at much lower than the "peak rate", Now the astronomers started using to infer the age of an individual galaxy from its colors (for a General explanation see [15]). Recent studies have shown that analyzing galaxy using tau models can give very erroneous estimates of star-formation rates and ages, and that adopting different models can improve the estimates. See Lee et al. 2009 [16]. Once the gas in a galaxy contains heavy elements, its ability to cool to form stars is dramatically altered. Most of the stars in any typical galaxy didn't form until billions of years later.

\subsubsection{Galaxies Born before Bigbang}

We know we can see Galaxies from 32 billion light-years distance, for example GNz-11. See Oesch, P. A. et al. [8] in their paper in March 2016 [8] observed a very bright at Galaxy at $z=11.1$ measured with $\mathrm{HST}$. This remote galaxy GN-z11, existed only 400 million years after the Big Bang, at a co-moving distance of 32 billion light years. We can see many instances and many Galaxies at very large distances as discussed in the introduction. Most probably these Galaxies are.

\subsubsection{Galaxies Born after Bigbang}

For example, let's consider few cases as discussed below. One can find scores of published papers on this subject.

Rakos, Schombert, and Odell in their paper "The Age of Cluster Galaxies from Continuum Colors" [17] determined the age of 1104 galaxies $(z=0.0046$ to 0.175 ) and find that galaxies in clusters can be separated into two groups, an old group with a mean age something similar to the age of Bigbang (12 Gyrs) and a much younger group with a mean age around 9 Gyrs. Here it may please be noted in both the groups deviations from the mean values are there. This can be easily visualized from their fig 1 , which shows ages, calculated using PCA method, are shown red are $>10$ Gyrs vs blue $<10$ Gyrs.

PAPOVICH et al. in their paper measured the sizes of compact passive galaxies [18], with their Sersic index and found that, at that redshift, in that cluster and around, these passive galaxies have smaller sizes than similar galaxies in the local universe. Hence these galaxies must have born in the subsequent $\sim 10$ billion years, and they are having an accelerated growth compared with Galaxies around.

\subsubsection{Early Massive Galaxies}

K. I. Caputi et al., in their paper in (2012) [19], have studied 25 extremely red $>4$ galaxies in the infrared emission ranges of gfalaxies at different cosmic distances from Spitzer Space Telescope and compared them with Hubble space telescope images also ( but very faint in the HST/CANDELS images), in the UKIDSS Ultra-Deep Survey field. The multi-wavelength analysis of these sources indicates that they are massive galaxies formed in the first two billion years. The observa- 
tions done in the last decade show that the most massive galaxies basically finished their growth about 8 billion years ago, while less massive galaxies continued forming stars even afterwards.

S. L. Finkelstein et al. in 2013 reported a Galaxy at redshift 7.51 and just 700 million years after Bigbang. They found the emission line at a wavelength of 1.0343 micrometres is likely to be Lyman a emission. This galaxy is having significant metal content, implying that galaxy become enriched rapidly with high star-formation rate of about 330 solar masses per year [20]. One can find many examples like this. This way these early massive Galaxies created the additional difficulty for the Bigbang LCDM based models.

\subsection{Galaxy Quenching}

According to White and Frenk (1991): the reproduction of the star populations of galaxies observed in the Universe requires significant energetic feedback to prevent over-cooling and excessive star formation. These words are applicable for some few Galaxies only within a LCDM cosmological paradigm. Process of changing of Star-forming spiral galaxies into elliptical galaxies is done through probably Feedback, which is a process that ejects gas (and metals) and energy into the ISM and IGM, which heats the gas and removes some it from the galaxy, which slows star formation.

Ruth Grützbauch et al. found: for a more highly evolved cluster XMMU J2235.3-57 at a redshift of 1.39. They described a "quenching radius", any galaxy within this distance from the cluster is extremely passive, but as you move further out the star formation rate increases.

The Mystery of Galactic Death: How Galaxies Quench: Faber et al. in 2007 [21] and Faber and Roger 2014 observed that, quenching happens in two ways: Fast track and another is Slow track. Slow-track quenching has two stages, first the central portions become gas poor as the sucked in halo gas reduces and this little amount gas that was sucked in cannot travel to go up to the center, and leaves a central hole. Here the central densemass of the Galaxy looses matter as radiation and does not get replenished. Slowly it leaves a hole at the Galaxy center, as the central densemass cannot hold the external stars together in the Galaxy. Most galaxies in the green valley at late times are on the slow track. In the second stage, these three processes "AGN feedback, morphological quenching, and galactic winds" act together to fade the outer disks. When the Galaxy mass is at transition mass of $3 \times 10^{10}$ solar masses the Galaxy may most probably headed for massive halo quenching. This can be visualized in most GV (Green valley) galaxies, see references for GV Galaxies [22] [23] whose inner parts have quenched. These are waiting for their disks to go out due to halo quenching. Total quenching time is several billion years. In Fast track the quenching galaxies quench in the classic merger-starburst-feedback model. Due to merger gas will come to center and triggers central starburst. In the first place the density of the inner parts will increase, outer disk destroyed. Total quenching time starting 
from merger to faded PSB (Post Starburst) Galaxies is about 0.5 to 1 billion years. Many such papers show that Galaxies will quench. For PSB (Post Star burst) Galaxies See the references [24] [25] [26].

\section{Shutting down Star Formation in Galaxies}

Faber et al. in 2007 [21] demonstrated by evaluating the luminosity function, shows that the density of red galaxies had a fourfold increase after $\mathrm{z}=1$, while density of blue galaxies was not changed. That means in some star forming galaxies, their star formation quenched and they advanced onto the red sequence, and at the same time new star forming galaxies were created.

\section{Part 2: Energy to Mass to Energy Cycle}

\subsection{Frequency Shift}

In Dynamic Universe Model this frequency shift happens on both the sides of spectrum. That means towards the frequency of a mass like neutrino or electron or positron also. In other words, Dynamic Universe Model predicts conversion of energy into mass. In accordance with Dynamic Universe Model this frequency shift happens on both the sides of spectrum. Here we will derive the results using general Physics and Mathematics that changes the frequency of electromagnetic radiation passing near a moving gravitating mass. The frequency of the radiation will increase (Blue shifted) when the relative movement of the gravitating body is in opposite direction to EM radiation and the frequency will reduce when in same direction (Red shifted). See the mathematical section for full understanding.

In accordance with Dynamic Universe Model frequency shift happens on both the sides of spectrum when any electromagnetic radiation passes grazingly near gravitating mass. This is a new fundamental prediction by Dynamic Universe Model, a foundational quest in the area of Astrophysics and Cosmology and a Grant is requested to verify this prediction. Here we will open a new frontier that will unlock way for formation of the basis for continual Nucleosynthesis (continuous formation of elements) in our Universe. For further information see the other papers published by the author. Here we will derive the results using general Newtonian Physics and Mathematics that changes the frequency of electromagnetic radiation passing near a moving gravitating mass. The frequency of the radiation will increase (Blue shifted) when the relative movement of the gravitating body is in opposite direction to EM radiation and the frequency will reduce when in same direction (Red shifted). This can be vice versa... Amount of frequency shift will depend on relative velocity difference.

Here the key AGENT involved is relative direction between Gravitating mass and radiation which will determine the frequency shift is either Red or Blue, i.e., the radiation frequency is either Blue shifted or Red shifted. Here in this project we will discuss about this AGENT involved in this process of frequency shifting in accordance with the prediction. This AGENT acts towards the frequency of a 
mass like neutrino, electron or positron also. In other-words Dynamic Universe Model predicts conversion of energy into mass.

Probably this AGENT will be the key for some fundamental difference between Blue shift and Red shift formations in addition to fundamental particle formation like Neutrinos, Electrons, Positrons which is the fundamental step for formation of other elements. We mean to say that this relative direction of motion of the electromagnetic ray passing grazingly near some huge mass, which causes the frequency shift will be the will fundamental difference between Blue shift and Red shift in astronomical observations.

All the other elements are formed in Stars, Novae, Super novae, Planet centers and Nebulae continually, and some of these elements will be destroyed to form energy like Sun and stars. This is the energy-mass-energy cycle. There were many papers describing these CONTINUAL elements FORMATION and transformation and change over, this is missing link or AGENT for these to complete the cycle.

\subsection{History of Frequency Shifting}

After 1922 Hubble published a series of papers in Astrophysical Journal describing various Galaxies and their red shifts/blue shifts. Using the new 100 inch Mt. Wilson telescope, Edwin Hubble was able to resolve the outer parts of some spiral nebulae as collections of individual stars and identified some Cepheid variables, thus allowing him to estimate the distance to the nebulae: they were far too distant to be part of the Milky Way. In the Ref. [8] [27] one can find more detailed analysis of this issue. And later using 200 inch Mt Palomar telescope Hubble could refine his search. In 1936 Hubble produced a classification system for galaxies that is used to this day, the Hubble sequence.

In the 1970s it was discovered in Vera Rubin's study of the rotation speed of gas in galaxies that the total visible mass (from the stars and gas) does not properly account for the speed of the rotating gas. This galaxy rotation problem is thought to be explained by the presence of large quantities of unseen dark matter. This dark matter question was discussed by Vera Rubin, see the Ref. [28] [29].

In fact there are millions of Blue shifted Galaxies not just 8300 found from 2009 by Hubble space telescope. "Beginning in the 1990s, the Hubble Space Telescope yielded improved observations. Among other things, Go to ADS search page try searching title and abstract with keywords "Blue shifted quasars". If you search with "and"s i.e., "Blue and Shifted and Galaxies" [use "and" option not with "or"option] you will find 248 papers in ADS search. I did not go through all of them. It established that the missing dark matter in our galaxy cannot solely consist of inherently faint and small stars. One can find more detailed analysis of this issue in the published ltarature on Hubble Deep Field, They gave an extremely long exposure of a relatively empty part of the sky, provided evidence that there are about 125 billion $\left(1.25 \times 10^{11}\right)$ galaxies in the un- 
iverse. Further details can be found at ref [8]. Improved technology in detecting the spectra invisible to humans (radio telescopes, infrared cameras, and $\mathrm{x}$-ray telescopes) allow detection of other galaxies that are not detected by Hubble. Particularly, galaxy surveys in the Zone of Avoidance (the region of the sky blocked by the Milky Way) have revealed a number of new galaxies. In the Ref. [18] one can find more detailed analysis of this issue.

Hubble Space Telescope's improved observational capabilities resolved as many as 8300 galaxies as Blue shifted till today which will discuss later in this paper.

\section{Part 3: Nucleosynthesis}

In Dynamic Universe Model the "light rays and other electromagnetic radiation" passing grazingly near any gravitating mass changes its frequency. This change in frequency will depend on relative direction of movement between mass and radiation. All these particles like "neutrinos, positrons, electrons, protons and neutrons" behave like waves also. We should remember the wave particle duality. Hence frequency enhancing is applicable here also. So in other words change in frequency can go further to converting radiation into matter like micro particles as stated above. Here in this paper we will discuss further into different element formations. And we will see some possible electrochemical reactions that are possible at high temperature and pressure for formation of these different elements.

\subsection{Frequency Shift}

General theory of relativity says that the frequency shift in electro-magnetic radiation near a gravitating mass happens in one direction only (Gravitational redshift). There in the EARLIER [30] paper we saw that Dynamic Universe Model says this frequency shift happens on both the sides of spectrum. That means towards the frequency of a mass like electron or positron or other particles also. In other words Dynamic Universe Model predicts conversion of energy into mass. We also should remember that though I am calling these as particles. All these particles like "neutrinos, positrons, electrons, protons and neutrons" behave like waves also. We should remember the wave particle duality. Hence frequency enhancing is applicable to these particles also.

Here in this paper we will further discuss formation of different elements. Main formation of (converting radiation) of photons of electromagnetic radiation into matter particles like particles of neutrinos, positrons, electrons, protons and neutrons was a slight enhancement of earlier paper. Such particles form Hydrogen or Helium. Some of these atoms will be attracted towards large gravitating masses like planets and stars. Remaining atoms which went far away will form Cosmic ray particles. That how there will be showers of Cosmic rays which we will discuss in the Cosmic ray formation section.

It may not be necessary to produce neutrons or neutrinos in initial stages. 
They can be created from some of the binding energy that was freed up as part of the fusion process. Proton creation would require that a $60 \mathrm{Mev}$ photon would have its frequency up shifted to about $3753 \mathrm{Mev}$ or close to 63 times its original frequency. So the shifting photon to proton would likely require a very large mass to make such a large frequency change in a single stroke. Or a CASCADE of stars can make this shift possible.

In a new star that has not converted much hydrogen into higher elements; the star is mostly composed of hydrogen. A basic hydrogen atom contains one proton and one electron. In the star the temperature is high enough to convert the atoms into plasma. Plasma is atoms with the electrons stripped off of from them. This means that the star contains free protons and free electrons. The first reaction is that two protons come together to form a nucleus. One of the protons decays into a neutron and a positron and neutrino which are both matter particles are given off as part of the process. This creates a deuterium atom, which is just a hydrogen atom that contains a neutron. This is what happens in SUN and Stars.

The next step is that the deuterium atom joins with another proton to form a Helium atom with just 1 neutron. A gamma ray energy photon is also given off because the energy required to bind the single Helium atom together is less than the energy of the separate proton and the deuterium atom combined. This gamma ray contains about $4 \mathrm{Mev}$ and comes completely from the savings in binding energy. The next step is for 2 of the above helium atoms to join together to produce a helium atom with 2 neutrons. The 2 extra protons are ejected. The protons carry off most of the freed binding energy about 15 to $20 \mathrm{Mev}$ in the form of kinetic energy or their motion. Energy released, higher atoms formed. Instead many hydrogen atoms have been combined together into fewer heavier atoms freeing up a small amount of energy in the process because the fewer heavier atoms require less total binding energy than the many more lighter hydrogen atoms required. For the most part, all of the matter is still there in the star it has just been compacted. It actually takes 4 hydrogen atoms (protons) to produce 1 helium atom. 2 of them are needed just as they are in the helium atom and the other 2 are converted into neutrons. The 4 protons contain a total of about $3753 \mathrm{Mev}$. The fusion reaction freed up about $60 \mathrm{MEV}$. In addition, all the heavier molecules upto uranium, are formed due to different nuclear reactions. There are many types of high energy particle bombardments that happen inside core of Stars, Cosmic ray bombardments and novae etc. The inside core of our own cool earth is not cool. Many elements are manufactured. The Novae and Supernovae and explosions of planets also contribute to different elements.

The other major elements are formed inside of stars; Magellanic Clouds, etc are formed as they are forming now, as explained by Hoyle. Those parts are not changed.

For the formation we require inside stars and planets. These star and planet centers will have the required high temperature and pressure. First of all we will 
discuss how mass gathers into these bigger stars and planets. Further sections will discuss about the electrochemical reactions required for formation of different elements.

The particles prepared in the process of energy conversion accumulate in the Magellanic Clouds or the star forming clouds or the dust regions of star forming Galaxies. If all the stars are formed during Bigbang why stars are formed again in a Galaxy?

\subsection{History of Nucleosynthesis}

The first steps of element formation were probably taken in 1920, by Arthur Eddington, who proposed that stars obtained their energy from nuclear fusion of hydrogen to form helium and raised the possibility that the heavier elements are produced in stars. In 1939, in a paper entitled "Energy Production in Stars", Hans Bethe analyzed the different possibilities for reactions by which hydrogen is fused into helium. He defined two processes that he believed to be the sources of energy in stars. The first one, the proton-proton chain reaction, is the dominant energy source in stars with masses up to about the mass of the Sun. The second process, the carbon-nitrogen-oxygen cycle, which was also considered by Carl Friedrich von Weizsäcker in 1938, is most important in more massive stars. These works concerned the energy generation capable of keeping stars hot.

The history of Big Bang nucleosynthesis began with the calculations of Ralph Alpher in the 1940s. Alpher published the Alpher-Bethe-Gamow paper that outlined the theory of light-element production in the early universe. During the 1970s, there was a major puzzle in that the density of baryons as calculated by Big Bang nucleosynthesis was much less than the observed mass of the universe based on calculations of the expansion rate. This puzzle was resolved in large part by postulating the existence of dark matter.

That theory was begun by Fred Hoyle in 1946 with his argument that a collection of very hot nuclei would assemble into iron. Hoyle followed that in 1954 with a large paper describing how advanced fusion stages within stars would synthesize elements between carbon and iron in mass. This is the dominant work in stellar nucleosynthesis. It provided the roadmap to how the most abundant elements on Earth had been synthesized from initial hydrogen and helium, making clear how those abundant elements increased their galactic abundances as the galaxy aged.

Quickly, Hoyle's theory was expanded to other processes, beginning with the publication of a celebrated review paper in 1957 by Burbidge, Burbidge, Fowler and Hoyle (commonly referred to as the B2FH paper). This review paper collected and refined earlier research into a heavily cited picture that gave promise of accounting for the observed relative abundances of the elements; but it did not itself enlarge Hoyle's 1954 picture for the origin of primary nuclei as much as many assumed, except in the understanding of nucleosynthesis of those elements heavier than iron. Significant improvements were made by Alastair GW 
Cameron and by Donald D. Clayton. [31] Cameron presented his own independent approach (following Hoyle's approach for the most part) of Nucleosynthesis. He introduced computers into time-dependent calculations of evolution of nuclear systems.

A clear physical description of the proton-proton chain and of the CNO cycle appears in a 1968 textbook by Clayton. For further details of the text book and papers see [31] [32] [33].

Bethe's two papers did not address the creation of heavier nuclei, however. Clayton calculated the first time-dependent models of the S-process and of the R-process, as well as of the burning of silicon into the abundant alpha-particle nuclei and iron-group elements, and discovered radiogenic chronologies for determining the age of the elements. The entire research field expanded rapidly in the 1970s.

\subsection{After Photon to Neutrino formation}

The view that the energy photons that are radiated from stars as a by product of the fusion of light elements such as hydrogen into helium would be changed back into more matter (presumably hydrogen) as it passes near large masses is an important one. This way the hydrogen; that is consumed in the universe will be remade and new stars will form says Dynamic Universe Model. This concept is different from Bigbang as it says all the hydrogen is produced at the time Bigbang, once it is consumed the star will die and similarly all the stars and the Universe will die out eventually.

To explain in other words, here the fusion of 2 hydrogen atoms into a helium atom occurs in a star, most of the mass or matter that was originally in the hydrogen atoms remains in the star in that helium atom. The helium atoms that are produced in that way can also fuse into heavier atoms and this process can continue up to iron. Iron and the atoms that are heavier than that are too close to the center of periodic table, the most stable point in the atomic scale to be able to fuse because it would actually take the addition of more energy to cause them to fuse than would be freed in the fusion reaction. When all of the lower elements have been fused, the end result is that most of the matter that was in those lighter elements, is now stored in the new midrange atoms that have been produced. The matter will be formed from energy only it is not from nothing. No matter will be formed from nothing in Dynamic Universe Model; the energy will change its form from one state to another only. The fusion and fission reactions will happen according to Atomic physics.

In my earlier paper I discussed how the Universe converts the energy to sub atomic particles. In this paper we will discuss the conversion particles into of hydrogen. But I still have not calculated whether that amount of hydrogen was equal to the amount hydrogen fuel burnt in the stars. But I feel that should be the case. Here what is happening is when all the stars die out, the whole Galaxy will quench. New Galaxies bourn some other place. I showed all the evidences 
regarding this in the paper titled "Distances, Locations, Ages and Reproduction of Galaxies in our Dynamic Universe" [34]. The Stars, Novae and Super Novae will convert all of the original hydrogen plus all of the newly produced midrange atoms that were produced by the fusion process. Some of the midrange elements and their compounds will be hosted by Planets, Comets, asteroids etc. So that there will NOT BE ANY OVERALL INCREASE in the total amount of matter in the universe. The Universe is not created from nothing. It sustains the matter and energy balance.

There are many astrophysical processes which can be responsible for Nucleosynthesis. Mostly they happen in stars. They can be listed as nuclear fusion processes are known as hydrogen burning (via the proton-proton chain or the CNO cycle), helium burning, carbon burning, neon burning, oxygen burning and silicon burning. These processes are able to create elements up to and including iron and nickel. Heavier elements can be assembled within stars by a neutron capture process known as the s-process or in explosive environments, such as supernovae, by a number of other processes. Some of those others include the r-process, which involves rapid neutron captures, the rp-process, and the p-process (sometimes known as the gamma process).

Abundances of the chemical elements in the Solar System: The relative abundance of elements won't change according to Dynamic Universe Model. The next three elements ( $\mathrm{Li}, \mathrm{Be}, \mathrm{B})$ are rare because they are poorly synthesized in stars. The two general trends in the remaining stellar-produced elements are: 1) an alternation of abundance of elements according to whether they have even or odd atomic numbers, and 2) a general decrease in abundance, as elements become heavier. Dynamic Universe Model Nucleosynthesis will result in mass abundances of about $75 \%$ of hydrogen-1, about $25 \%$ helium- 4 , about $0.01 \%$ of deuterium and helium-3, trace amounts (on the order of 10-10) of lithium, and negligible heavier elements.

Those particles which are negatively charged will be attracting positive particles nearby due to electro-static attraction to make neutral particles or some may get converted back to lower frequencies. All these particles will have their own frequencies due to wave particle duality. Generally these radiation or particles will have very high speeds comparable to that of light.

The slower particles form clouds of interstellar medium or inter-galaxy medium observed in the Galaxies and in the Universe. From these clouds and dust higher accumulations are known are star forming clouds or AGN (Active Galactic Nuclei), which forms stars.

What happens in the core or other levels in a star depends mostly on its size and mass. Small stars less than $1 / 2$ the size of the sun can only fuse hydrogen because they do not have enough mass to generate the pressure and temperature needed to fuse helium. Stars of the size of the sun can fuse hydrogen and helium, but can't fuse any larger atoms, as an example. Each time a heavier element is fused in a star it gives off less energy than the fusion of the previous next smaller 
atom. To say it in a different way, when you fuse helium atoms you get less energy freed up by the fusion process than you would when you fuse hydrogen atoms. You can still get some freed up energy by fusing atoms up to iron. Large stars actually do fuse iron, but they do that just before they explode in a supernova. When they fuse the iron it takes more energy to fuse it than is freed up, so the net effect is to cool the core, etc. When the core cools it cannot resist the pull of gravity, so it collapses. The end result is the supernova explosion. The fusion of iron and the lighter elements can produce elements up to about zinc by various processes. The larger elements are generally considered to be mostly made in the supernova explosions, etc.

That large mass would be exerting a great pull on the photon and it would likely pull the photon into itself before it could generate that large a frequency change. Some the photons would be escaping. It would be Blackhole to attract all the photons into it. But Blackholes are not there according to Dynamic Universe Model.

The light photon travelling at velocity of light will eventually escapes the inward pull of the large star. Because of its high velocity even it would then be subjected to the continual pull of the large mass as it travelled away from it and that pull would then WOUN'T downshift the photon's frequency again back to the $60 \mathrm{Mev}$ it had at the start. So some the photons which are formed thro passing a cascade of stars or passing by a large star will somehow converted into the protons and electrons while its frequency was still up shifted. These matter particles would not lose their speed immediately, but will lose their velocity close to light slowly. Hence these protons electrons positrons etc will lose motion as and becomes part of that some mass. It will not be the starting larger mass. They need not be pulled back into the large mass that had up-shifted the photon.

So it would likely be NOT only very large stars or Blackholes, etc. that would have any chance of producing that large of a frequency up-shift, but a CASCADE of stars also do the same thing. As there are no Blackholes, the only choices left are other two only. And the new matter particles that would which are left and not pulled into stars or planets are lost will become Cosmic rays. Those particles which or pulled into the small stars or large stars and fused along with its other hydrogen.

Lets observe and see. We have to first observe that there will be frequency shift and is happening. If it is not, there can be a fundamental mistake. But I don't think. The frequency shift is happening. We have yet to observe it. Many predictions of Dynamic Universe model came true. Lets see this. For me I never saw thro' a telescope till now. I don't have any access, I am not rich also to buy such equipment. I am just a theoretician.

Most of the heavier elements especially those up near and including uranium are generally not fused in stars as a normal part of their fusion process. Even large stars cannot generate the pressure and temperature needed to produce 
these heavy atoms. They are mostly produced in supernova explosions, etc. The earth is much too small in mass to generate the pressure and temperature necessary to even fuse hydrogen into helium.

With the formation of stars, heavier nuclei were created from hydrogen and helium by stellar nucleosynthesis, a process that continues today. Some of these elements, particularly those lighter than iron, continue to be delivered to the interstellar medium when low mass stars eject their outer envelope before they collapse to form white dwarfs. The remains of their ejected mass form the planetary nebulae observable throughout our galaxy.

The process of conversion from energy into partices, is a continuous process. Electromagnetic radiation energy is created in stars. This radiation will be passing grazingly to some gravitating mass and will be converted back continuously into elementary particles at various gravitating masses. Slowly these various particles change into higher massive particles or may get bombarded into stars or planets. Here in this paper the formation of elements is divided into 6 processes. They are Elementary particles and elements generated in frequency changing process, By Cosmic rays, By Small stars, By Large Stars, By Super Novae and Manmade elements By Neutron Stars. This paper discusses mainly about first two categories. The other processes are standard processes.

There are many astrophysical processes which can be responsible for Nucleosynthesis. Mostly they happen in stars. They can be listed as nuclear fusion processes are known as hydrogen burning (via the proton-proton chain or the CNO cycle), helium burning, carbon burning, neon burning, oxygen burning and silicon burning. These processes are able to create elements up to and including iron and nickel. Heavier elements can be assembled within stars by a neutron capture process known as the s-process or in explosive environments, such as supernovae, by a number of other processes. Some of those others include the r-process, which involves rapid neutron captures, the rp-process, and the p-process (sometimes known as the gamma process). These process are well defined in wikipedia dynamic Universe model does not propose any changes in these. Only in the radiation passing grazingly near stars will have its frequency changed so that matter will be formed.

\subsection{Elementary Particles and Elements Periodic Table}

These are generated in frequency changing process. These are particles like "neutrinos, positrons, electrons, protons and neutrons etc" are used for generating during Nucleosynthesis for forming the elements hydrogen, helium, nuclei of hydrogen, nuclei of helium and alpha particles etc. This process is widespread, happens in the entire universe. Hence the production of positive ions of the simplest isotope of hydrogen $1 \mathrm{H}$, which is nothing but a single proton is done in the element formation process near cascade of stars or near large stars. See the Figure 1 for the details of other elements formation. 


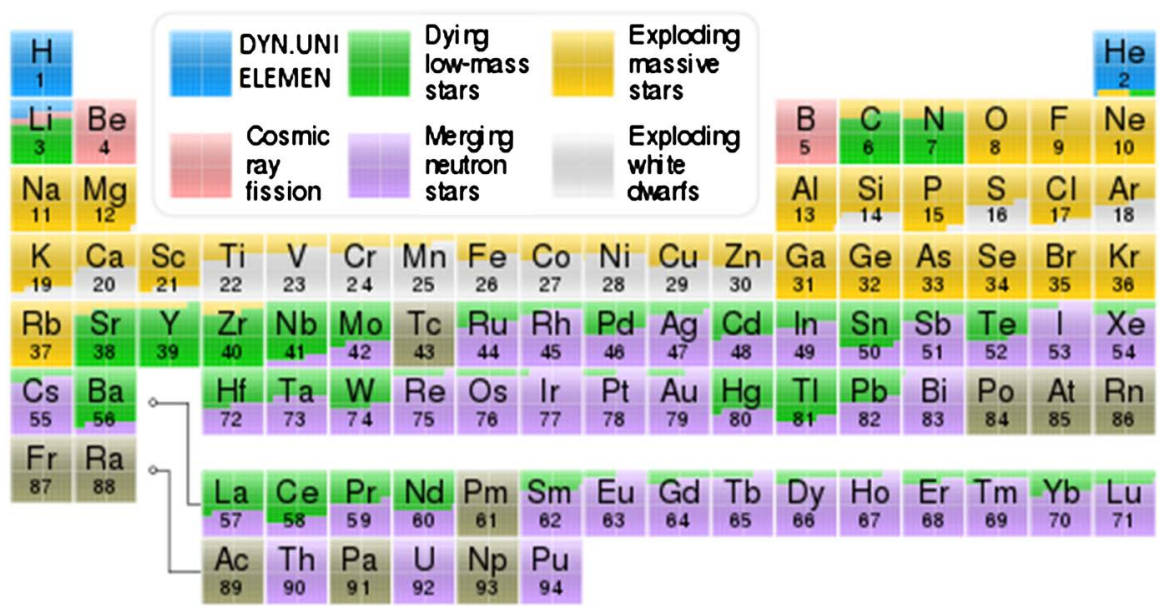

Figure 1. A periodic table indicating the origins of elements including elements formed by Dynamic Universe Model frequency shifting is shown here. All elements above 94 are not incorporated which are including manmade elements. This table shows the elements made according this paper shown as DYN.UNI ELEMEN. This figure is originally from Wikipedia's Nucleosynthesis, and it was modified according to our paper here. This figure gives bird's eye view of this whole paper.

\subsubsection{By Cosmic Rays}

The elements lithium, barium and boron come under this category. We can see these elements are prepared in stellar Nucleosynthesis. Some of the particles in the universe will escape to the boundary of universe and will be attracted back into universe and such particles will become cosmic ray showers. Cosmic ray spallation, caused when cosmic rays impact the interstellar medium and fragment larger atomic species, is a significant source of the lighter nuclei, particularly ${ }^{3} \mathrm{He},{ }^{9} \mathrm{Be}$ and ${ }^{10,11} \mathrm{~B}$, that are not created by stellar nucleosynthesis. Cosmic ray bombardment of elements on Earth also contribute to the presence of rare, short-lived atomic species called cosmogenic nuclides

Cosmic ray spallation process reduces the atomic weight of interstellar matter by the impact with cosmic rays, to produce some of the lightest elements present in the universe (though not a significant amount of deuterium). Most notably spallation is believed to be responsible for the generation of almost all of ${ }^{3} \mathrm{He}$ and the elements lithium, beryllium, and boron. The spallation process results from the impact of cosmic rays (mostly fast protons) against the interstellar medium. These impacts fragment carbon, nitrogen, and oxygen nuclei present. The process results in the light elements beryllium, boron, and lithium in cosmos at much greater abundances than they are within solar atmospheres. The light elements ${ }^{1} \mathrm{H}$ and ${ }^{4} \mathrm{He}$ nuclei are not a product of spallation and are represented in the cosmos with approximately primordial abundance. Beryllium and boron are not significantly produced by stellar fusion processes, since ${ }^{8} \mathrm{Be}$ is not particle-bound.

Due to Cosmic ray bombardments in proton-rich regions of the Magellanic clouds lithium-6, beryllium-9, and boron-11 could have been produced in the reactions: 


$$
\begin{gathered}
{ }^{4} \mathrm{He}+{ }^{2} \mathrm{H} \rightarrow{ }^{6} \mathrm{Li}+\gamma \\
{ }^{7} \mathrm{Li}+{ }^{3} \mathrm{H} \rightarrow{ }^{9} \mathrm{Be}+\mathrm{n} \\
{ }^{7} \mathrm{Li}+{ }^{2} \mathrm{H} \rightarrow{ }^{9} \mathrm{Be}+\gamma \\
{ }^{7} \mathrm{Be}+{ }^{3} \mathrm{H} \rightarrow{ }^{9} \mathrm{Be}+\mathrm{p} \\
{ }^{9} \mathrm{Be}+{ }^{3} \mathrm{H} \rightarrow{ }^{11} \mathrm{~B}+\mathrm{n}
\end{gathered}
$$

A star gains heavier elements by combining its lighter nuclei, hydrogen, deuterium, beryllium, lithium, and boron, which were found in the initial composition of the interstellar medium and hence the star. Interstellar gas therefore contains declining abundances of these light elements. Larger quantities of these lighter elements in the present universe are therefore thought to have been restored through billions of years of cosmic ray (mostly high-energy proton) mediated breakup of heavier elements in interstellar gas and dust. The fragments of these cosmic-ray collisions include the light elements $\mathrm{Li}, \mathrm{Be}$ and $\mathrm{B}$.

\subsubsection{Stellar Nucleosynthesis: The Proton-Proton Chain Reaction}

In 1939, Hans Bethe [35] proposed that one of the protons could decay by beta emission into a neutron via the weak interaction during the brief moment of fusion, making deuterium a vital product in the chain. This idea was part of the body of work in stellar nucleosynthesis for which Bethe won the Nobel Prize in Physics in 1967.

The first step is fusion of two ${ }^{1} \mathrm{H}$ nuclei (protons) into deuterium, releasing a positron and a neutrino as one proton changes into a neutron. It is a two-stage process; first, two protons fuse to form a diproton:

$$
{ }_{1}^{1} \mathrm{H}+{ }_{1}^{1} \mathrm{H} \rightarrow{ }_{2}^{2} \mathrm{He}+\gamma
$$

followed by the beta-plus decay of the diproton to deuterium:

$$
{ }_{2}^{2} \mathrm{He} \rightarrow{ }_{1}^{2} \mathrm{H}+\mathrm{e}^{+}+\mathrm{v}_{\mathrm{e}}
$$

with the overall formula:

$$
{ }_{1}^{1} \mathrm{H}+{ }_{1}^{1} \mathrm{H} \rightarrow{ }_{1}^{2} \mathrm{H}+\mathrm{e}^{+}+\mathrm{v}_{\mathrm{e}}+\gamma
$$

This first step is extremely slow because the positron emission of the diproton to deuterium is extremely rare (the vast majority of the time, the diproton decays back into hydrogen-1 through proton emission). We will have to work our further to have some value of the time of combination... But one can see the paper by Bethe (1939) for further reference for some more details. [35] This is because the emission of the positron is brought about by the weak nuclear force, which is immensely weaker than the strong nuclear force and the electromagnetic force.

$$
\mathrm{e}^{-}+\mathrm{e}^{+} \rightarrow 2 \gamma+1.02 \mathrm{MeV}
$$

The deuterium fuses with another proton to produce the light isotope of helium, ${ }^{3} \mathrm{He}$ : 


$$
{ }_{1}^{2} \mathrm{D}+{ }_{1}^{1} \mathrm{H} \rightarrow{ }_{2}^{3} \mathrm{He}+\gamma+5.49 \mathrm{MeV}
$$

This process, mediated by the strong nuclear force rather than the weak force, is extremely fast by comparison to the first step.

From here there are four possible paths to generate ${ }^{4} \mathrm{He}$.

In the Sun, ${ }^{4} \mathrm{He}$ synthesis via branch p-p I occurs with a frequency of 83.30 percent, p-p II with 16.68 percent, and p-p III with 0.02 percent. There is also the extremely rare p-p IV branch. Other even-rarer reactions may occur. The rate of these reactions is very low due to very small cross-sections, or because the number of reacting particles is so low that any reactions that might happen are statistically insignificant. This is partly why no mass- 5 or mass- 8 elements are seen. While the reactions that would produce them, such as a proton + helium -4 producing lithium-5, or two helium-4 nuclei coming together to form beryllium-8, may actually happen, these elements are not detected because there are no stable isotopes of atomic masses 5 or 8 ; the resulting products immediately decay into their initial reactants.

The overall reaction is:

$$
4 \mathrm{p} \rightarrow{ }^{4} \mathrm{He}+2 \mathrm{e}^{+}+2 v_{\mathrm{e}}
$$

\subsubsection{The P-P I Branch}

$$
{ }_{2}^{3} \mathrm{He}+{ }_{2}^{3} \mathrm{He} \rightarrow{ }_{2}^{4} \mathrm{He}+2{ }_{1}^{1} \mathrm{H}+12.86 \mathrm{MeV}
$$

The complete p-p I chain reaction releases a net energy of $26.732 \mathrm{MeV}$. Two percent of this energy is lost to the neutrinos that are produced. The p-p I branch is dominant at temperatures of 10 to $14 \mathrm{MK}$. Below $10 \mathrm{MK}$, the P-P chain does not produce much ${ }^{4} \mathrm{He}$.

\subsubsection{The P-P II Branch}

Proton-proton II chain reaction for Lithium processing. The P-P II branch is dominant at temperatures of 14 to $23 \mathrm{MK}$.

$$
\begin{aligned}
{ }_{2}^{3} \mathrm{He}+{ }_{2}^{4} \mathrm{He} \rightarrow{ }_{4}^{7} \mathrm{Be}+\gamma \\
{ }_{4}^{7} \mathrm{Be}+\mathrm{e}^{-} \rightarrow{ }_{3}^{7} \mathrm{Li}+v_{\mathrm{e}}+0.861 \mathrm{MeV} / 0.383 \mathrm{MeV} \\
{ }_{3}^{7} \mathrm{Li}+{ }_{1}^{1} \mathrm{H} \rightarrow 2{ }_{2}^{4} \mathrm{He}
\end{aligned}
$$

Note that the energies in the equation above are not the energy released by the reaction. Rather, they are the energies of the neutrinos that are produced by the reaction. 90 percent of the neutrinos produced in the reaction of ${ }^{7} \mathrm{Be}$ to ${ }^{7} \mathrm{Li}$ carry an energy of $0.861 \mathrm{MeV}$, while the remaining 10 percent carry $0.383 \mathrm{MeV}$.

\subsubsection{The P-P III Branch}

The Proton-proton III chain reaction are given below.

$$
\begin{gathered}
{ }_{2}^{3} \mathrm{He}+{ }_{2}^{4} \mathrm{He} \rightarrow{ }_{4}^{7} \mathrm{Be}+\gamma \\
{ }_{4}^{7} \mathrm{Be}+{ }_{1}^{1} \mathrm{H} \rightarrow{ }_{5}^{8} \mathrm{~B}+\gamma
\end{gathered}
$$




$$
\begin{gathered}
{ }_{5}^{8} \mathrm{~B} \rightarrow{ }_{4}^{8} \mathrm{Be}+\mathrm{e}^{+}+v_{\mathrm{e}} \\
{ }_{4}^{8} \mathrm{Be} \rightarrow 2{ }_{2}^{4} \mathrm{He}
\end{gathered}
$$

The P-P III chain is dominant if the temperature exceeds $23 \mathrm{MK}$. The p-p III chain is not a major source of energy in the Sun (only 0.11 percent), but it was very important in the solar neutrino problem because it generates very high energy neutrinos (up to $14.06 \mathrm{MeV}$ ).

\subsubsection{The P-P IV (Hep) Branch}

This reaction is predicted theoretically, but it has never been observed due to its rarity (about $0.3 \mathrm{ppm}$ in the Sun). In this reaction, helium- 3 captures a proton directly to give helium-4, with an even higher possible neutrino energy (up to $18.8 \mathrm{MeV}$ ).

$$
{ }_{2}^{3} \mathrm{He}+{ }_{1}^{1} \mathrm{H} \rightarrow{ }_{2}^{4} \mathrm{He}+\mathrm{e}^{+}+v_{\mathrm{e}}+18.8 \mathrm{MeV}
$$

\subsubsection{The PEP Reaction}

\section{Proton-proton and electron-capture chain reactions in a star}

Deuterium can also be produced by the rare pep (proton-electron-proton) reaction (electron capture):

$$
{ }_{1}^{1} \mathrm{H}+\mathrm{e}^{-}+{ }_{1}^{1} \mathrm{H} \rightarrow{ }_{1}^{2} \mathrm{D}+v_{\mathrm{e}}
$$

Both the pep and p-p reactions can be seen as two different Feynman representations of the same basic interaction, where the electron passes to the right side of the reaction as an antielectron. This is represented in the figure of proton-proton and electron-capture chain reactions in a star, available at the NDM'06 web site.

\subsection{Stellar Nucleosynthesis}

The most important reactions in stellar Nucleosynthesis are, Hydrogen fusion, Deuterium fusion, The proton-proton chain, The carbon-nitrogen-oxygen cycle, Helium fusion, The triple-alpha process, The alpha process etc.

For Fusion of heavier elements: Lithium burning: a process found most commonly in brown dwarfs, Carbon-burning process, Neon-burning process, Oxygen-burning process, Silicon-burning process etc...

Production of elements heavier than iron: Neutron capture, The R-process, The S-process, Proton capture, The Rp-process, Photo-disintegration, The P-process etc...

\subsubsection{By Small Stars}

Carbon, Nitrogen, Oxygen, Neon and sulphur are synthesised by small stars, but these can be produced by some medium and bigger stars also. Atomic numbers less than Iron are here. The first direct proof that nucleosynthesis occurs in stars was the astronomical observation that interstellar gas has become enriched with heavy elements as time passed. As a result, stars that were born from it late in the galaxy, formed with much higher initial heavy element abundances than those 
that had formed earlier. Stellar nucleosynthesis creates these elements: small amounts of the hydrogen isotope deuterium $(2 \mathrm{H}$ or $\mathrm{D})$, the helium isotope helium-3 $\left({ }^{3} \mathrm{He}\right)$, and a very small amount of the lithium isotope lithium-7 $\left({ }^{7} \mathrm{Li}\right)$. In addition to these stable nuclei, two unstable or radioactive isotopes were also produced: the heavy hydrogen isotope tritium ( $3 \mathrm{H}$ or $\mathrm{T})$; and the beryllium isotope beryllium-7 ( $\left.{ }^{7} \mathrm{Be}\right)$; but these unstable isotopes later decayed into ${ }^{3} \mathrm{He}$ and ${ }^{7} \mathrm{Li}$, as above. Essentially all of the elements that are heavier than Hydrogen were created by stellar nucleosynthesis in evolving and exploding stars.

Neutrons can react with positrons or electron neutrinos to create protons and other products in one of the following reactions:

$$
\begin{gathered}
\mathrm{n}+\mathrm{e}^{+} \leftrightarrow \operatorname{anti}_{-} v_{\mathrm{e}}+\mathrm{p} \\
\mathrm{n}+v_{\mathrm{e}} \leftrightarrow \mathrm{p}+\mathrm{e}^{-}
\end{gathered}
$$

For comparison sake, Big Bang nucleosynthesis (BBN) produced NO elements heavier than lithium, due to a bottleneck: the absence of a stable nucleus with 8 or 5 nucleons. Hence this makes BBN redundant and not required. In stars, the bottleneck is passed by triple collisions of helium-4 nuclei, producing carbon (the triple-alpha process). However, this process is very slow, taking tens of thousands of years to convert a significant amount of helium to carbon in stars.

The subsequent nucleosynthesis of the heavier elements requires the extreme temperatures and pressures found within stars and supernovas. Star formation has occurred continuously in the galaxy since that time. The elements found on Earth, the so-called primordial elements, were created prior to Earth's formation by stellar nucleosynthesis and by supernova nucleosynthesis. They range in atomic numbers from $Z=6$ (carbon) to $Z=94$ (plutonium). Synthesis of these elements occurred either by nuclear fusion (including both rapid and slow multiple neutron capture) or to a lesser degree by nuclear fission followed by beta decay.

Stellar nucleosynthesis is the nuclear process by which new nuclei are produced. It occurs in stars during stellar evolution. It is responsible for the galactic abundances of elements from carbon to iron. Stars are thermonuclear furnaces in which $\mathrm{H}$ and $\mathrm{He}$ are fused into heavier nuclei by increasingly high temperatures as the composition of the core evolves. Of particular importance is carbon, because its formation from $\mathrm{He}$ is a bottleneck in the entire process. Carbon is produced by the triple-alpha process in all stars. Carbon is also the main element that causes the release of free neutrons within stars, giving rise to the s-process, in which the slow absorption of neutrons converts iron into elements heavier than iron and nickel.

The products of stellar nucleosynthesis are generally dispersed into the interstellar gas through mass loss episodes and the stellar winds of low mass stars. The obvious reason is the is Gas is most targeted place for the newly formed particles.

Deuterium is in some ways the opposite of helium- 4 in that while helium- 4 is 
very stable and very difficult to destroy, deuterium is only marginally stable and easy to destroy. The temperatures, time, and densities were sufficient to combine a substantial fraction of the deuterium nuclei to form helium-4 but insufficient to carry the process further using helium- 4 in the next fusion step.

Deuterium fusion, also called deuterium burning, is a nuclear fusion reaction that occurs in stars and some substellar objects, in which a deuterium nucleus and a proton combine to form a helium-3 nucleus. It occurs as the second stage of the proton-proton chain reaction, in which a deuterium nucleus formed from two protons fuses with another proton.

Deuterium is the most easily fused nucleus available to accreting protostars, and such fusion in the center of protostars can proceed when temperatures exceed $106 \mathrm{~K}$. The reaction rate is so sensitive to temperature that the temperature does not rise very much above this. The energy generated by fusion drives convection, which carries the heat generated to the surface.

If there was no deuterium fusion, there would be no stars with masses more than about two or three times the mass of the Sun in the pre-main-sequence phase as the more intense hydrogen fusion would occur and prevent the object from accreting matter. Deuterium fusion allows further accretion of mass by acting as a thermostat that temporarily stops the central temperature from rising above about one million degrees, a temperature not hot enough for hydrogen fusion, but allowing time for the accumulation of more mass. When the energy transport mechanism switches from convective to radiative, energy transport slows, allowing the temperature to rise and hydrogen fusion take over in a stable and sustained way. Hydrogen fusion will begin at $107 \mathrm{~K}$.

Overview of the CNO-I cycle. The helium nucleus is released at the top-left step.

Hydrogen fusion (nuclear fusion of four protons to form a helium-4 nucleus is the dominant process that generates energy in the cores of main-sequence stars. There are two predominant processes by which stellar hydrogen fusion occurs: proton-proton chain and the carbon-nitrogen-oxygen (CNO) cycle. Ninety percent of all stars, with the exception of white dwarfs, are fusing hydrogen by these two processes.

In the cores of lower-mass main-sequence stars such as the Sun, the dominant energy production process is the proton-proton chain reaction. This creates a helium-4 nucleus through a sequence of chain reactions that begin with the fusion of two protons to form a deuterium nucleus (one proton plus one neutron) along with an ejected positron and neutrino. In each complete fusion cycle, the proton-proton chain reaction releases about $26.2 \mathrm{MeV}$. The proton-proton chain reaction cycle is relatively insensitive to temperature; a $10 \%$ rise of temperature would increase energy production by this method by $46 \%$, hence, this hydrogen fusion process can occur in up to a third of the star's radius and occupy half the star's mass. For stars above 35\% of the Sun's mass, the energy flux toward the surface is sufficiently low and energy transfer from the core region remains by 
radiative heat transfer, rather than by convective heat transfer. As a result, there is little mixing of fresh hydrogen into the core or fusion products outward.

\subsubsection{By Large Stars}

The detection of technetium in the atmosphere of a red giant star in 1952, by spectroscopy, provided the first evidence of nuclear activity within stars. Because technetium is radioactive, with a half-life much less than the age of the star, its abundance must reflect its recent creation within that star. Equally convincing evidence of the stellar origin of heavy elements, is the large overabundances of specific stable elements found in stellar atmospheres of asymptotic giant branch stars. Observation of barium abundances some 20 - 50 times greater than found in unevolved stars is evidence of the operation of the s-process within such stars.

Many modern proofs of stellar nucleosynthesis are provided by the isotopic compositions of stardust, solid grains that have condensed from the gases of individual stars and which have been extracted from meteorites. Stardust is one component of cosmic dust, and is frequently called presolar grains. The measured isotopic compositions in stardust grains demonstrate many aspects of nucleosynthesis within the stars from which the grains condensed during the star's late-life mass-loss episodes.

Hydrogen fusion requires much higher temperatures and pressures than does deuterium fusion, hence, there are objects massive enough to burn deuterium but not massive enough to burn hydrogen. These objects are called brown dwarfs, and have masses between about 13 and 80 times the mass of Jupiter. Brown dwarfs may shine for a hundred million years before their deuterium supply is burned out.

Objects above the deuterium-fusion minimum mass (deuterium burning minimum mass, DBMM) will fuse all their deuterium in a very short time $(\sim 4$ $50 \mathrm{Myr}$ ), whereas objects below that will burn little, and hence, preserve their original deuterium abundance. "The apparent identification of free-floating objects, or rogue planets below the DBMM would suggest that the formation of star-like objects extends below the DBMM."

In higher-mass stars, the dominant energy production process is the $\mathrm{CNO}$ cycle, which is a catalytic cycle that uses nuclei of carbon, nitrogen and oxygen as intermediaries and in the end produces a helium nucleus as with the proton-proton chain. During a complete $\mathrm{CNO}$ cycle, $25.0 \mathrm{MeV}$ of energy is released. The difference in energy production of this cycle, compared to the proton-proton chain reaction, is accounted for by the energy lost through neutrino emission. The CNO cycle is very temperature sensitive, a $10 \%$ rise of temperature would produce a $350 \%$ rise in energy production. About $90 \%$ of the CNO cycle energy generation occurs within the inner $15 \%$ of the star's mass, hence it is strongly concentrated at the core. The core region becomes a convection zone, which stirs the hydrogen fusion region and keeps it well mixed with the surrounding proton-rich region. This core convection occurs in stars where the CNO cycle contributes more than $20 \%$ of the total energy. As the star ages and 
the core temperature increases, the region occupied by the convection zone slowly shrinks from $20 \%$ of the mass down to the inner $8 \%$ of the mass. Our Sun produces $10 \%$ of its energy from the CNO cycle.

The type of hydrogen fusion process that dominates in a star is determined by the temperature dependency differences between the two reactions. The proton-proton chain reaction starts at temperatures about $4 \times 106 \mathrm{~K}$, making it the dominant fusion mechanism in smaller stars.

The Triple-alpha process and Alpha process: Main sequence stars accumulate helium in their cores as a result of hydrogen fusion, but the core does not become hot enough to initiate helium fusion. Helium fusion first begins when a star leaves the red giant branch after accumulating sufficient helium in its core to ignite it. In stars around the mass of the sun, this begins at the tip of the red giant branch with a helium flash from a degenerate helium core and the star moves to the horizontal branch where it burns helium in its core. Cepheid variables fuse helium until the core is largely carbon and oxygen. The most massive stars become supergiants when they leave the main sequence and quickly start helium fusion as they become red supergiants. After helium is exhausted in the core of a star, it will continue in a shell around the carbon-oxygen core.

In all cases, helium is fused to carbon via the triple-alpha process. This can then form oxygen, neon, and heavier elements via the alpha process. In this way, the alpha process preferentially produces elements with even numbers of protons by the capture of helium nuclei. Elements with odd numbers of protons are formed by other fusion pathways.

\subsubsection{By Super Novae}

Supernova nucleosynthesis occurs in the energetic environment in supernovae, in which the elements between silicon and nickel are synthesized in quasiequilibrium established during fast fusion that attaches by reciprocating balanced nuclear reactions to $28 \mathrm{~S}$. From aluminium silicon to Uranium, Neptunium and plutonium. Many elements in this list are produced by large stars also. Supernova nucleosynthesis within exploding stars by fusing carbon and oxygen is responsible for the abundances of elements between magnesium (atomic number 12) and nickel (atomic number 28). This is also responsible for the creation of rarer elements heavier than iron and nickel, and uranium and thorium, in the last few seconds of a type II supernova event. The synthesis of these heavier elements absorbs energy as they are created, from the energy produced during the supernova explosion.

\subsubsection{Explosive Nucleosynthesis}

The r-process, rp-process, and Supernova nucleosynthesis etc.

Quasiequilibrium can be thought of as almost equilibrium except for a high abundance of the 28Si nuclei in the feverishly burning mix. This concept was the most important discovery in nucleosynthesis theory of the intermediate-mass elements since Hoyle's 1954 paper because it provided an overarching under- 
standing of the abundant and chemically important elements between silicon (A $=28)$ and nickel $(A=60)$. It replaced the incorrect although much cited alpha process of the $\mathrm{B}^{2} \mathrm{FH}$ paper, which inadvertently obscured Hoyle's better 1954 theory. Further nucleosynthesis processes can occur, in particular the r-process (rapid process) described by the $\mathrm{B}^{2} \mathrm{FH}$ paper and first calculated by Seeger, Fowler and Clayton, in which the most neutron-rich isotopes of elements heavier than nickel are produced by rapid absorption of free neutrons. The creation of free neutrons by electron capture during the rapid compression of the supernova core along with assembly of some neutron-rich seed nuclei makes the r-process a primary process, and one that can occur even in a star of pure $\mathrm{H}$ and He. This is in contrast to the $\mathrm{B}^{2} \mathrm{FH}$ designation of the process as a secondary process. This promising scenario, though generally supported by supernova experts, has yet to achieve a totally satisfactory calculation of r-process abundances. The primary r-process has been confirmed by astronomers who have observed old stars born when galactic metallicity was still small, that nonetheless contain their complement of r-process nuclei; thereby demonstrating that the metallicity is a product of an internal process. The r-process is responsible for our natural cohort of radioactive elements, such as uranium and thorium, as well as the most neutron-rich isotopes of each heavy element. The rp-process (rapid proton) involves the rapid absorption of free protons as well as neutrons, but its role and its existence are less certain.

Explosive nucleosynthesis occurs too rapidly for radioactive decay to decrease the number of neutrons, so that many abundant isotopes with equal and even numbers of protons and neutrons are synthesized by the silicon quasiequilibrium process. During this process, the burning of oxygen and silicon fuses nuclei that themselves have equal numbers of protons and neutrons to produce nuclides which consist of whole numbers of helium nuclei, up to 15 (representing 60Ni). Such multiple-alpha-particle nuclides are totally stable up to $40 \mathrm{Ca}$ (made of 10 helium nuclei), but heavier nuclei with equal and even numbers of protons and neutrons are tightly bound but unstable. The quasiequilibrium produces radioactive isobars $44 \mathrm{Ti}, 48 \mathrm{Cr}, 52 \mathrm{Fe}$, and $56 \mathrm{Ni}$, which (except $44 \mathrm{Ti}$ ) are created in abundance but decay after the explosion and leave the most stable isotope of the corresponding element at the same atomic weight. The most abundant and extant isotopes of elements produced in this way are $48 \mathrm{Ti}, 52 \mathrm{Cr}$, and $56 \mathrm{Fe}$. These decays are accompanied by the emission of gamma-rays (radiation from the nucleus), whose spectroscopic lines can be used to identify the isotope created by the decay. The detection of these emission lines were an important early product of gamma-ray astronomy.

The most convincing proof of explosive nucleosynthesis in supernovae occurred in 1987 when those gamma-ray lines were detected emerging from supernova SN 1987A. Gamma ray lines identifying 56Co and 57Co nuclei, whose radioactive half-lives limit their age to about a year, proved that they were created by their radioactive cobalt parents. This nuclear astronomy observation 
was predicted in 1969 as a way to confirm explosive nucleosynthesis of the elements, and that prediction played an important role in the planning for NASA's Compton Gamma-Ray Observatory.

Other proofs of explosive nucleosynthesis are found within the stardust grains that condensed within the interiors of supernovae as they expanded and cooled. Stardust grains are one component of cosmic dust. In particular, radioactive $44 \mathrm{Ti}$ was measured to be very abundant within supernova stardust grains at the time they condensed during the supernova expansion. This confirmed a 1975 prediction of the identification of supernova stardust (SUNOCONs), which became part of the pantheon of pre-solar grains. Other unusual isotopic ratios within these grains reveal many specific aspects of explosive nucleosynthesis.

\subsubsection{Manmade Elements}

The last elements in the periodical table are produced artificially. A few minor natural processes continue to produce very small numbers of new nuclides on Earth. These nuclides contribute little to their abundances, but may account for the presence of specific new nuclei.

Very small amounts of certain nuclides are produced on Earth by artificial means. Those are our primary source, for example, of technetium. However, some nuclides are also produced by a number of natural means that have continued after primordial elements were in place.

Radioactive decay may lead to radiogenic daughter nuclides. The nuclear decay of many long-lived primordial isotopes, especially uranium-235, uranium-238, and thorium-232 produce many intermediate daughter nuclides, before they too finally decay to isotopes of lead. The Earth's natural supply of elements like radon and polonium is via this mechanism. The atmosphere's supply of argon-40 is due mostly to the radioactive decay of potassium-40 in the time since the formation of the Earth. Little of the atmospheric argon is primordial. Helium-4 is produced by alpha-decay, and the helium trapped in Earth's crust is also mostly non-primordial. In other types of radioactive decay, such as cluster decay, larger species of nuclei are ejected (for example, neon-20), and these eventually become newly formed stable atoms.

Radioactive decay may lead to spontaneous fission. This is not cluster decay, as the fission products may be split among nearly any type of atom. Thorium-232, uranium-235, and uranium-238 are primordial isotopes that undergo spontaneous fission. Natural technetium and promethium are produced in this manner.

Nuclear reactions: Naturally-occurring nuclear reactions powered by radioactive decay give rise to so-called nucleogenic nuclides. This process may also cause the production of further subatomic particles, such as neutrons. Neutrons can also be produced in spontaneous fission and by neutron emission. These neutrons can then go on to produce other nuclides via neutron-induced fission, or by neutron capture. For example, some stable isotopes such as neon-21 and neon-22 are produced by several routes of nucleogenic synthesis, and thus only 
part of their abundance is primordial.

Nuclear reactions due to cosmic rays. By convention, these reaction-products are not termed "nucleogenic" nuclides, but rather cosmogenic nuclides. Cosmic rays continue to produce new elements on Earth by the same cosmogenic processes discussed above that produce primordial beryllium and boron. One important example is carbon-14, produced from nitrogen-14 in the atmosphere by cosmic rays. Iodine-129 is another example.

\subsubsection{Neutron Star Collision}

In addition to artificial processes, it is postulated that neutron star collision is the main source of elements heavier than iron. Neutron star collisions occur in a fashion similar to Type Ia supernovae. When two neutron stars orbit each other closely, they spiral inward as time passes. They may colloid with each other. This creates a magnetic field that is trillions of times stronger than that of Earth, in a matter of one or two milliseconds. Astronomers believe that this event is what creates certain kinds of gamma-ray bursts.

This completes some general introduction about formation of all the elements in the Universe without any Big bang and using only Dynamic Universe model, Now lets see what is this Dynamic Universe Model ...

\section{About Dynamic Universe Model}

\subsection{UGF (Universal Gravitational Force)}

In our Dynamic Universe every mass is moving in a direction and goal determined universal gravitational force (UGF) as the indomitable resultant vector of gravitational forces acted by all the other bodies in the Universe. This UGF is not constant force acting in only one direction. This resultant UGF vector force is varying according to ever varying dynamic movements and positions of all the masses in the Universe from time to time. In Dynamic Universe Model, this UGF is the fundamental concept; this model calculates this force "UGF" from moment to moment using its mathematical laws on each and every mass in the SITA simulations. In this way many present-day unsolved physics problems were solved. This method is different from conventional two body problem solution [36].

Dynamic Universe Model is a singularity free tensor-based math model. The tensors used are linear without using any differential or integral equations. Only one calculated output set of values exists. Data means properties of each point mass like its three-dimensional coordinates, velocities, accelerations and it's mass. Newtonian two-body problem used differential equations.

This universe exists now in the present state, it existed earlier, and it will continue to exist in future also in a similar way. All physical laws will work at any time and at any place. Here in this dynamic universe, both the red shifted and blue shifted Galaxies co-exist simultaneously.

SITA solution can be used in many places like presently unsolved applications 
like Pioneer anomaly at the Solar system level, Missing mass due to Star circular velocities and Galaxy disk formation at Galaxy level, and prediction of blue shifted Galaxies.

This Model is new Cosmological model fundamentally and mathematically different from Bigbang, Steady state model etc. Dynamic Universe Model is based on laws of Newtonian dynamics. It is a simple multi-body problem solution. This Dynamic Universe Model is a singularity free and body-body collision free n-body problem solution based on UGF acting on each and every body with some mass in the Universe. In this model "time" moves in one direction i.e. into future only. 133 masses were used in this setup, and the "same setup" was used for last 25 years on a simple PC without any problem in its SITA simulations. Its SITA simulations can solve many unsolved cosmological problems and successfully published solutions to vast variety of present day scientific problems. The calculations can be done over the particle's entire path throughout time. These calculations can go into future for making predictions. With this model, it was found with uniform mass distribution in space, the masses will colloid. But there are no singularities. With non-uniform mass densities, the masses trend to rotate about each other after some time-steps and they don't colloid. SITA is a simple computer implementable solution of Dynamic Universe Model. Its many predictions came true like existence Blueshifted Galaxies in the universe. Its prediction that there is no dark-matter was experimentally proved later. (Many papers and books were published by the author, Details are available at [37]).

\subsection{Main Foundational Points of Dynamic Universe Model}

No Isotropy; No Homogeneity; No Space-time continuum; Non-uniform density of matter(Universe is lumpy); No singularities; No collisions between bodies; No Blackholes; No warm holes; No Bigbang; No repulsion between distant Galaxies; Non-empty Universe; No imaginary or negative time axis; No imaginary $\mathrm{X}, \mathrm{Y}, \mathrm{Z}$ axes; No differential and Integral Equations mathematically; No General Relativity and Model does not reduce to General Relativity on any condition; No Creation of matter like Bigbang or steady-state models; No many mini Bigbangs; No Missing Mass; No Dark matter; No Dark energy; No Bigbang generated CMB detected; No Multi-verses etc.

\section{Mathematical Background}

The mathematics of Dynamic Universe Model is published and is available in many open access papers hence not repeated here. The following linear tensor Equation (1) is the basis for all these calculations.

$$
\Phi_{\text {ext }}(\alpha)=-\sum_{\substack{\beta=1 \\ \alpha \neq \beta}}^{N^{\gamma}} \frac{G m_{\beta}^{\gamma}}{\left|x^{\gamma \beta}-x^{\gamma \alpha}\right|}-\sum_{\substack{\beta=1 \\ \alpha \neq \beta}}^{N^{\delta \gamma}} \frac{G m_{\beta}^{\delta \gamma}}{\left|x^{\delta \gamma \beta}-x^{\delta \gamma \alpha}\right|}
$$

This concept can be extended to still higher levels in a similar way. There are other variations of the mathematics used in some different applications like 
VLBI explanations. The above Equation (1) is the main resourceful equation, which gives many results that are not possible otherwise today.

\subsection{Derivation of Equations for the Effect of Movement of Gravitational Mass on the Frequency of the Incoming Light Ray with c}

The rest mass of the photon is $=m=E / c^{2}$. Gravitational field of the mass (Sun or star or some gravitational mass) $=g_{o}$. The distance of the photon from center $=$ $r$. Energy $=E g_{o} r / c^{2}$. Frequency of photon $=\mathcal{\vartheta}=E / h$ or $E=h \vartheta$.

Case 1. When the velocity of gravitational mass is opposite to the incoming light ray:

In this case the gravitational field will act as some brake on the incoming light ray.

The gravitating mass is moving with a velocity $\mu$ in the opposite direction and applies brake on the photon. This is something similar to the case where the gravitational mass is fixed in position and the photon of the rest mass $E / c^{2}$ is moving with velocity $\mu+c$

Hence the initial velocity of photon $=-\mu-c$. It's velocity is towards the gravitational mass. The photon is having a freefall. Its final velocity $=-\mu-c-g_{o} t$ [ where $t$ is the time of flight of photon].

$$
\begin{aligned}
& \text { Initial Energy }=m(\mu+c)^{2} / 2=E(\mu+c)^{2} / 2 c^{2}=E\left(\mu^{2}+c^{2}+2 \mu c\right) / 2 c^{2} \\
& \qquad \begin{aligned}
\text { Final Energy } & =\frac{1}{2}\left(E / c^{2}\right)\left(-\mu-c-g_{o} t\right)^{2} \\
& =\frac{1}{2}\left(E / c^{2}\right)\left(\mu^{2}+c^{2}+g_{o}^{2} t^{2}+2 \mu g_{o} t+2 c g_{o} t+2 \mu c\right)
\end{aligned}
\end{aligned}
$$

Change in Energy $=\frac{1}{2}\left(E / c^{2}\right)\left(g_{o}^{2} t^{2}+2 \mu g_{o} t+2 c g_{o} t\right)$, here $E=h \vartheta$ that means

$$
\text { Change in Energy }=\frac{1}{2}\left(h \vartheta / c^{2}\right)\left(g_{o}^{2} t^{2}+2 \mu g_{o} t+2 c g_{o} t\right)
$$

Hence

$$
\text { change in Frequency }=\vartheta=1 /\left\{2\left(h / c^{2}\right)\left(g_{o}^{2} t^{2}+2 \mu g_{o} t+2 c g_{o} t\right)\right\}
$$

Here the frequency increases. The incoming ray from a distant Galaxy will be Red shifted.

Case 2. When the velocity of gravitational mass is same direction as the incoming light ray.

In this case the gravitational field will enhance the energy of the incoming light ray.

The gravitating mass is moving with a velocity $\mu$ in the same direction and enhances energy of the photon. This is something similar to the case where the gravitational mass is fixed in position and the photon of the rest mass $E / c^{2}$ is moving with velocity $(c-\mu)$

Hence the initial velocity of photon $=(c-\mu)$. It's velocity is towards the gra- 
vitational mass. The photon is having a freefall. Its final velocity $=-\mu+c-g_{o} t$ [where $t$ is the time of flight of photon].

$$
\begin{aligned}
& \text { Initial Energy }=m(-\mu+c)^{2} / 2=E(-\mu+c)^{2} / 2 c^{2}=E\left(\mu^{2}+c^{2}-2 \mu c\right) / 2 c^{2} \\
& \begin{aligned}
\text { Final Energy } & =\frac{1}{2}\left(E / c^{2}\right)\left(-\mu+c-g_{o} t\right)^{2} \\
& =\frac{1}{2}\left(E / c^{2}\right)\left(\mu^{2}+c^{2}+g_{o}^{2} t^{2}+2 \mu g_{o} t-2 c g_{o} t-2 \mu c\right)
\end{aligned}
\end{aligned}
$$

Change in Energy $=\frac{1}{2}\left(E / c^{2}\right)\left(g_{o}^{2} t^{2}+2 \mu g_{o} t-2 c g_{o} t\right)$, here $E=h \vartheta$ that means

$$
\text { Change in Energy }=\frac{1}{2}\left(h \vartheta / c^{2}\right)\left(g_{o}^{2} t^{2}+2 \mu g_{o} t-2 c g_{o} t\right)
$$

Hence

$$
\text { change in Frequency }=\vartheta=1 /\left\{2\left(h / c^{2}\right)\left(g_{o}^{2} t^{2}+2 \mu g_{o} t-2 c g_{o} t\right)\right\}
$$

Here the frequency decreases. Incoming ray from a distant Galaxy will be Blue shifted.

Case 3. When the velocity of gravitational mass is not exactly opposite or exactly in the same direction to the incoming light ray.

In this case the gravitational field will act as some brake or enhance the energy of the incoming light ray depending on $(\cos \phi)$ of the velocity of gravitational mass relative to incoming radiation, where $(\phi)$ is the angle between the light ray and velocity of gravitational mass.

The gravitating mass is moving with a velocity $\mu$ in the opposite direction and applies brake on the photon. This is something similar to the case where the gravitational mass is fixed in position and the photon of the rest mass $E / c^{2}$ is moving with velocity $\mu \cos \phi+c$

Hence the initial velocity of photon $=-\mu \cos \phi-c$. It's velocity is towards the gravitational mass. The photon is having a freefall. Its final velocity $=-\mu-c-$ $g_{o} t$ [where $t$ is the time of flight of photon].

$$
\begin{aligned}
\text { Initial Energy } & =m(\mu \cos \phi+c)^{2} / 2=E(\mu \cos \phi+c)^{2} / 2 c^{2} \\
& =E\left(\mu^{2} \cos ^{2} \phi+c^{2}+2 \mu \cos \phi c\right) / 2 c^{2}
\end{aligned}
$$

Final Energy $=\frac{1}{2}\left(E / c^{2}\right)\left(-\mu \cos \phi-c-g_{o} t\right)^{2}$

$$
=\frac{1}{2}\left(E / c^{2}\right)\left(\mu^{2} \cos ^{2} \phi+c^{2}+g_{o}^{2} t^{2}+2 \mu \cos \phi g_{o} t+2 c g_{o} t+2 \mu \cos \phi c\right)
$$

Change in Energy $=\frac{1}{2}\left(E / c^{2}\right)\left(g_{o}^{2} t^{2}+2 \mu \cos \phi g_{o} t+2 c g_{o} t\right)$, here $E=h \mathcal{\vartheta}$ that means

$$
\text { Change in Energy }=\frac{1}{2}\left(h \vartheta / c^{2}\right)\left(g_{o}^{2} t^{2}+2 \mu \cos \phi g_{o} t+2 c g_{o} t\right)
$$

Hence

$$
\text { change in Frequency }=\vartheta=1 /\left\{2\left(h / c^{2}\right)\left(g_{o}^{2} t^{2}+2 \mu \cos \phi g_{o} t+2 c g_{o} t\right)\right\}
$$


Here it can be observed that Equation (34) is the main equation and the Equations ((32) and (33)) are special cases of Equation (34). It will become Equation (32) when $\phi$ is " 0 degrees" and Equation (33) when $\phi$ is 180 degrees.

\subsection{Input Data}

We simulated a Galaxy named GNz11 and was placed at a distance of 100 times 13.4 Billion light years (1.26862E +28 meters). 132 more galaxies were assumed in the range of distances $10^{25}$ to $10^{28}(3.02001 \mathrm{E}+26$ to $1.26862 \mathrm{E}+28)$ meters. The masses were also in the range of Galaxy masses. We allowed the simulation system on self-gravitation calculations for 102 Iterations. Then we reduced first Galaxy distance by 50\%. Calculations were done for next 100 iterations. We did another set of calculations without reducing the distance of first Galaxy for the comparison.

\subsection{Starting Graphs and Last Iteration Graphs}

For better visualization the graphs are further sub divided to show 33 Galaxies each (these graphs were not shown here due to page length constraint), in addition to all Galaxies in one single graph. A set of first 10 Galaxies were also shown in the first set of graphs to see the difference in positions more clearly. All the graphs are XY graphs. Here in the first column all the graphs are pertaining to the simulations where GNz11 is taken in its assumed distance. And in the second column all the graphs are pertaining to the simulations where GNz11 is taken as $50 \%$ of its assumed distance and in the names a word DC was added to indicate that Distance was Changed. The graphs showing the starting positions are named Start and the graphs showing last iterations are named Last in the graph names.

From the end of last iteration graphs as shown in the Table 1 and the Table 2 the Universe looks the same. See the paper for the reference for full details. That means the distance changing of simulated Galaxy GNz11 has no effect on the shape of the Universe on the large scales.

\section{Conclusions}

From the end of last iteration graphs as shown in the Table 1 the Universe looks the same. That means the distance changing of simulated Galaxy GNz11 has no effect on the shape of the Universe on the large scales. That means the distance changing has no effect on overall shape of the Universe. That means Galaxies can be at any distance as decided by the Dynamic Equilibrium of UGF (Universal Gravitation force) in this model ...

This paper shows the evidences by the way of earlier published papers. The Galaxy GN-z11, born only 400 million years after the Big Bang, exists at a distance of 32 billion light years. Bigbang explains these by co-moving distance. If we don't accept Bigbang then how will you explain these Galaxies at these huge distances? Galaxies born 6 billion years after Bigbang, and massive Galaxies born 300 million years immediately after Bigbang. Many of Galaxies were born about 
Table 1. Contains graphs of starting positions and last positions of first 10 Galaxies used in this simulation that is including GNz11. Only these graphs are shown here due to page length constraint and to visualize the exact differences between all these 4 graphs. On can very easily see that the graph named " 1 to $10 \mathrm{DC}$ start XY" is different from all the others, which is showing the starting position of GNz11changed by 50\%. And also it may please be noted that SCALES in these start graphs are different to accommodate more clear view.

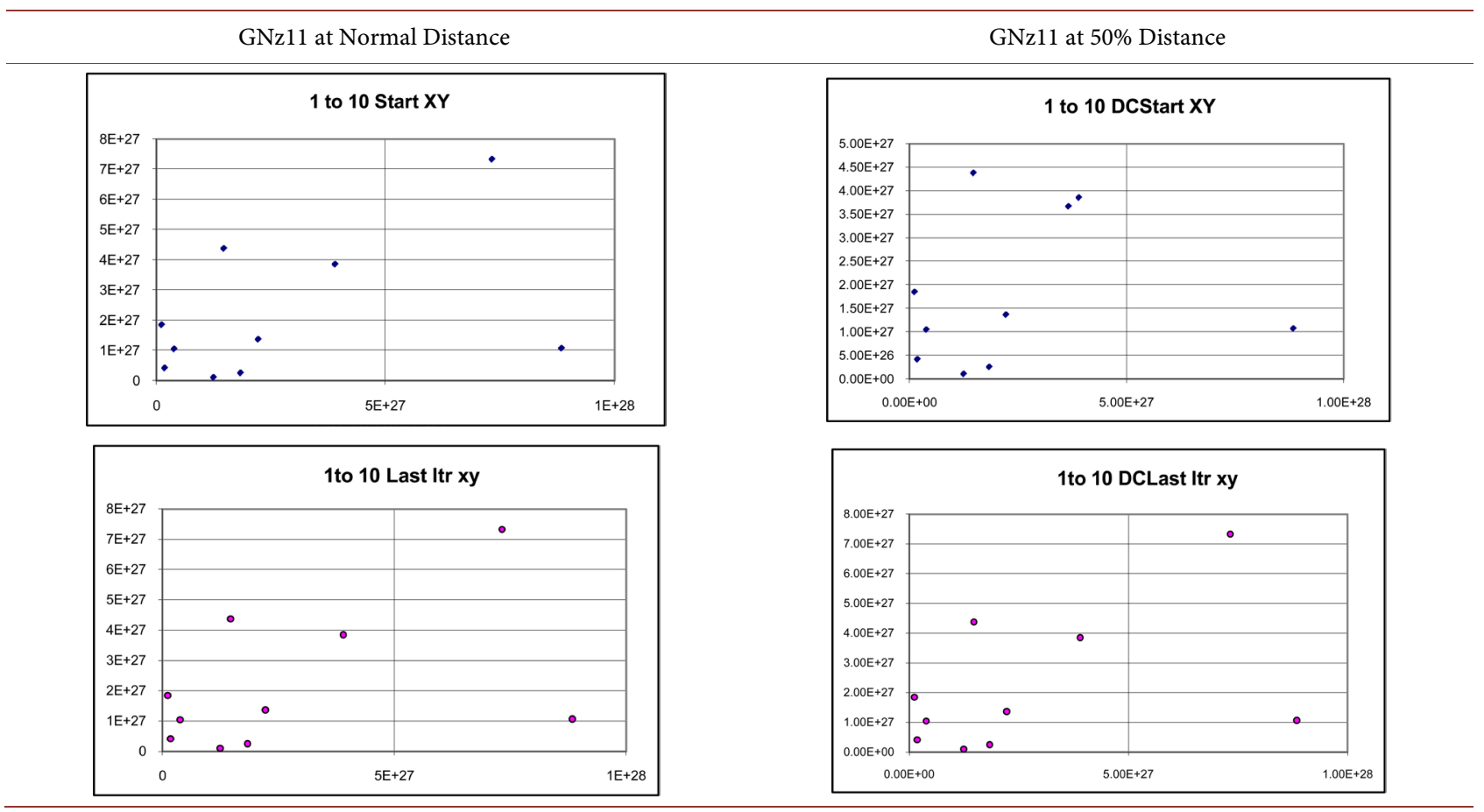

Table 2. Contains graphs of starting positions and last positions of first 33 Galaxies used in this simulation that is including GNz11. These graphs are for visualizing the exact differences between all these 4 graphs. On can very easily see that the graph named " 1 to 33 DC start XY" is different from all the others, which is showing the starting position of GNz11changed by $50 \%$.

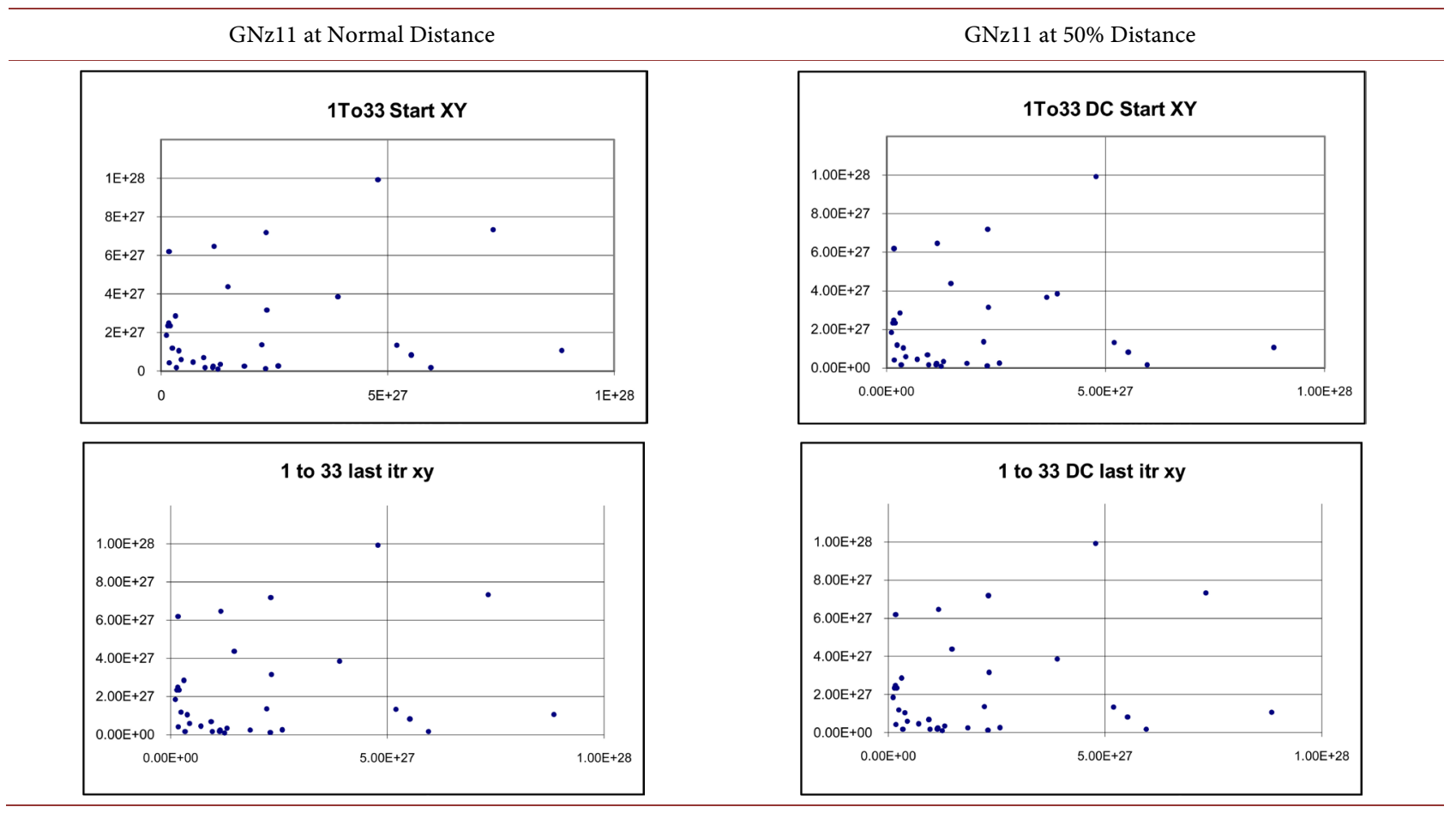


3 billion years after Bigbang. The present thinking of Bigbang is about 13.8 billion years with the popular $\lambda \mathrm{CDM}$ models. Galaxy quenching is happening.

By seeing all these, the author proposes a new idea that the Universe had an ability to reproduce Galaxies. Here probably the "biological world" learned from the Universe and subsequently the physical systems learnt to pursue the goal of reproduction and formed the "Biological world". Slowly these biological life forms acquired intelligence and are now trying to understand Universe! That way probably the goal-oriented behavior is a physical or cosmological trend of the Universe ...

Let's see what other conclusions I can made from other parts of the paper.

The particles are prepared in the process of energy conversion accumulate in the Magellanic Clouds or the star forming clouds or the dust regions of star forming Galaxies. If all the stars are formed during Bigbang why stars are formed again in a Galaxy?

The elementary particles are generated in frequency changing process. Here we have shown that all the elements are being prepared by Cosmic rays, by Small stars, by Large Stars, by Super Novae, Manmade elements and by Neutron Stars.

Dynamic Universe model is based on hard observed facts and gives many verifiable facts. Even though the frequency shifting as described here was not a physically observed fact, the earlier [30] paper gives possible outcome viz., blue and red shifting.

We also discussed how all the elements are formed...

For a serious reader we gave some additional references for further study... see references [39]-[57].

\section{Acknowledgements}

I sincerely thank mother of all mothers "VAK" for continuously guiding this research. I am very much thankful for the cooperation by my wife Savitri.

\section{Conflicts of Interest}

The author declares no conflicts of interest regarding the publication of this paper.

\section{References}

[1] Gupta, S.N.P. (2012) SITA: Dynamic Universe Model: Blue Shifted Galaxies Prediction. Lap Publications, Saarbrucken.

[2] Gupta, S.N.P. (2017) Nucleosynthesis after Frequency Shifting in Electromagnetic Radiation near Gravitating Masses in Dynamic Universe Model with Math.

[3] Einstein, A. (1952) The Foundation of General Theory of Relativity. Dover Publications, New York.

[4] Gupta, S.N.P. (2011) Dynamic Universe Model: SITA Singularity Free Software. VDM Publications, Saarbrucken.

[5] Gupta, S.N.P. (2011) Dynamic Universe Model: SITA Software Simplified. VDM 
Publications, Saarbrucken.

[6] GUPTA, S.N.P. (2015) Dynamic Universe Model Predicts the Live Trajectory of New Horizons Satellite Going To Pluto. Applied Physics Research, 7, 63.

[7] Encyclopaedia Britannica. https://www.britannica.com/biography/Jagadish-Chandra-Bose

[8] Oesch, P.A., Brammer, G., van Dokkum, P., et al. (2016) A Remarkably Luminous Galaxy at $\mathrm{z}=11.1$ Measured with Hubble Space Telescope Grism Spectroscopy. The Astrophysical Journal, 819, 129. arXiv:1603.00461. https://doi.org/10.3847/0004-637X/819/2/129

[9] Zitrin, A., Labbe, I., et al. (2015) Lyman-Alpha Emission from a Luminous z = 8.68 Galaxy: Implications for Galaxies as Tracers of Cosmic Reionization. arXiv:1507.02679v3 [astro-ph.GA]

[10] Oesch, P.A., van Dokkum, P.G., et al. (2015) A Spectroscopic Redshift Measurement for a Luminous Lyman Break Galaxy at $Z=7: 730$ Using Keck/Mosfire. arXiv:1502.05399v2 [astro-ph.GA]

[11] http://www.gsjournal.net/Science-Journals/Research\%20Papers-Astrophysics/Down load/6932 http://vixra.org/pdf/1703.0263v1.pdf

[12] https://ned.ipac.caltech.edu/level5/March15/Kennicutt/Kennicutt5.html Star Formation in the Galaxy, an Observational Overview-IfA, Hawaii www.ifa.hawaii.edu/ reipurth/reviews/lada_yukawa.pdf by C.J. Lada-The Problems of Star and Planet Formation Are among the Most Important Challenges ... Support of the Concept of Present Epoch Galactic star formation.

[13] Star formation.pdf https://www.astro.umd.edu/ richard/ASTRO421/star\%20formation.pdf

[14] http://candels-collaboration.blogspot.in/2012/08/the-multi-wavelength-shapes-of-g alaxies.html and Another Paper Is ... Seong-Kook Lee1, The Astrophysical Journal Supplement Series, 184:100-132, 2009 September doi:10.1088/0067-0049/184/1/100 "Biases and Uncertainties in Physical Parameter Estimates of Lyman Break Galaxies From Broadband Photometry". http://iopscience.iop.org/article/10.1088/0067-0049/184/1/100/pdf

[15] http://candels-collaboration.blogspot.in/2012/08/the-multi-wavelength-shapes-of-g alaxies.html

[16] Lee, S.-K. (2009) Biases and Uncertainties in Physical Parameter Estimates of Lyman Break Galaxies from Broadband Photometry. The Astrophysical Journal Supplement Series, 184, 100-132.

http://iopscience.iop.org/article/10.1088/0067-0049/184/1/100/pdf https://doi.org/10.1088/0067-0049/184/1/100

[17] Rakos, Schombert and Odell (2008) The Age of Cluster Galaxies from Continuum Colors. The Astrophysical Journal, 677, 1019. https://doi.org/10.1086/533513

[18] Papovich, C., et al. (2012) Candels Observations of the Structural Properties of Cluster Galaxies at $Z=1.62$. https://arxiv.org/pdf/1110.3794v2.pdf

[19] Caputi, K.I., et al. (2012) The Nature of Extremely Red $H$ - [4.5] > 4 Galaxies Revealed with Seds and Candels. The Astrophysical Journal Letters, 750, L20.

[20] Finkelstein, S.L., et al. (2013) A Galaxy Rapidly Forming Stars 700 Million Years after the Big Bang at Redshift 7.51. Nature letters, 502, 524-527. https://doi.org/10.1038/nature12657

[21] Faber, S.M., et al. (2007) Galaxy Luminosity Functions to $\mathrm{z} \sim 1$ from DEEP2 and 
COMBO-17: Implications for Red Galaxy Formation. The Astrophysical Journal, 665, 265-294. https://doi.org/10.1086/519294

[22] Nuclear Activity Is More Prevalent in Star-Forming Galaxies ... http://iopscience.iop.org/article/10.1088/0004-637X/771/1/63/pdf PDF file Driver et al. 2006; Kauffmann et al. 2006; Franx et al. Published in: The Astrophysical Journal, 2013.

[23] Quenching Depends on Morphologies: Implications from the Ultraviolet ... http://www.oalib.com/paper/3582477 Green Valley (GV) Galaxies with the Galaxy Evolution Explorer (GALEX)+Sloan Digital Sky Survey (SDSS).

[24] Post-Starburst Galaxies: The Missing Link in Galaxy ... "Post-Starburst Galaxies: The Missing Link in Galaxy Evolution", Joanna Ramasawmy, Feb 2018|Daily Paper Summaries.

https://astrobites.org/2018/02/01/post-starburst-galaxies-the-missing-link-in-galaxy

[25] Galaxies on the Road to Quenching - unipd.it http://paduaresearch.cab.unipd.it/10221/1/Paccagnella_Angela_tesi.pdf PDF File Galaxies on the Road to Quenching Direttore della scuola di dottorato: Prof. GiampaoloPiotto Supervisore: Dott. Bianca MariaPoggianti Correlatore: Dott. ... (PSB) galaxies ...

[26] Galaxy And Mass Assembly (GAMA): The Mechanisms for Quiescent Galaxy ... https://researchbank.swinburne.edu.au/file/3b953b8a-34e7-4f29-82a2-2f1de9565630 /1/2018-rowlands-galaxy_and_mass.pdf PDF File, "Galaxy And Mass Assembly (GAMA): The Mechanisms for Quiescent Galaxy Formation at $\mathrm{z}<1$ " Rowlands, Wild et al. 2016).

[27] Shi, X. and Turner, M.S. (1998) Expectations for the Difference between Local and Global Measurements of the Hubble Constant. Astrophysics Journal, 493, 519-522. https://doi.org/10.1086/305169

[28] Rubin, V.C. (1983) Dark Matter in Spiral Galaxies. Scientific American, 248, 96-106. https://doi.org/10.1038/scientificamerican0683-96

[29] Rubin, V.C. (2000) One Hundred Years of Rotating Galaxies. Publications of the Astronomical Society of the Pacific, 112, 747-750. https://doi.org/10.1086/316573

[30] Gupta, S.N.P. (2018) Energy to Mass Conversion in Dynamic Universe Model. SciFed Journal of Nuclear Science, 2, 15.

[31] Clayton, D.D. (1968) Principles of Stellar Evolution and Nucleosynthesis. University of Chicago Press, Chicago.

[32] Bodansky, D., Clayton, D.D. and Fowler, W.A. (1968) Nucleosynthesis during Silicon Burning. Physical Review Letters, 20, 161-164. https://doi.org/10.1103/PhysRevLett.20.161

[33] Bodansky, D., Clayton, D.D. and Fowler, W.A. (1968) Nuclear Quasi-Equilibrium during Silicon Burning. The Astrophysical Journal Supplement Series, 16, 299. https://doi.org/10.1086/190176

[34] Gupta, S.N.P. (2017) Distances, Locations, Ages and Reproduction of Galaxies in Our Dynamic Universe by Satyavarapu Naga Parameswara Gupta. FQXi Essay Contest, 2016-2017, http://fqxi.org/community/forum/topic/2726

[35] Bethe, H.A. (1939) Physical Review, 55, 103, 434; cited in Donald D. Clayton, Principles of Stellar Evolution and Nucleosynthesis, The University of Chicago Press, $1983,366$.

[36] http://vaksdynamicuniversemodel.blogspot.in/2012/11/fundamental-questions-add $\underline{\text { ressed-by.html }}$ 
[37] SNP. Gupta All My Papers and Books Available at http://vaksdynamicuniversemodel.blogspot.in/p/10-feb-201-6-all-my-published-pa pers.html http://vaksdynamicuniversemodel.blogspot.in/p/books-published.html

[38] Schawinski, K., et al. (2014) The Green Valley Is a Red Herring: Galaxy Zoo Reveals Two Evolutionary Pathways towards Quenching of Star Formation in Early- and Late-Type Galaxies? Monthly Notices of the Royal Astronomical Society, 000, 1-20.

[39] Bouwens, R., Bradley, L., et al. (2014) A Census of Star-Forming Galaxies in the z 9-10 Universe Based on HST+Spitzer Observations Over 19 CLASH Clusters: Three Candidate $\mathrm{z} \sim 9-10$ Galaxies and Improved Constraints on the Star Formation Rate Density at z 9. The Astrophysical Journal, 795, 126. https://doi.org/10.1088/0004-637X/795/2/126

[40] Gupta, S.N.P., Murty, J.V.S. and Krishna, S.S.V. (2014) Mathematics of Dynamic Universe Model Explain Pioneer Anomaly. Nonlinear Studies USA, 21, 26-42.

[41] Tully, R.B. and Fisher, J.R. (1987) Nearby Galaxies Atlas. Cambridge University Press, Cambridge.

[42] Gupta, S.N.P. (2013) Introduction to Dynamic Universe Model. International Journal of Scientific Research and Reviews Journal, 2, 203-226.

[43] Aguirre, A. and Gratton, S. (2002) Steady-State Eternal Inflation. Physical Review $D, 65$, Article ID: 083507. https://doi.org/10.1103/PhysRevD.65.083507

[44] Gupta, S.N.P. (2015) “No Dark Matter" Prediction from Dynamic Universe Model Came True! Journal of Astrophysics and Aerospace Technology, 3, Article ID: 1000117.

[45] Gupta, S.N.P. (2014) Dynamic Universe Model's Prediction “No Dark Matter” in the Universe Came True! Applied Physics Research, 6, 8-25.

[46] Bouwens, R., Bradley, L., et al. (2014) "A Census of Star-Forming Galaxies in the z 9-10 Universe Based on HST+Spitzer Observations over 19 CLASH clusters:" arXiv:1211.2230v3 [astro-ph.CO]

[47] Some Good Wikipedia Pages. https://en.wikipedia.org/wiki/Proton-proton_chain https://en.wikipedia.org/wiki/CNO_cycle https://en.wikipedia.org/wiki/Helium_fusion https://en.wikipedia.org/wiki/Carbon_burning_process https://en.wikipedia.org/wiki/Neon_burning_process https://en.wikipedia.org/wiki/Oxygen_burning_process https://en.wikipedia.org/wiki/Silicon_burning_process https://en.wikipedia.org/wiki/S-process https://en.wikipedia.org/wiki/Supernova https://en.wikipedia.org/wiki/R-process https://en.wikipedia.org/wiki/Rp-process https://en.wikipedia.org/wiki/P-process

[48] Francesco, P. and Hans, Z. (2002) Physics of Star Formation in Galaxies. Springer-Verlag, Berlin, 21-25.

[49] John, B. and Bo, R. (2006) The Birth of Stars and Planets. Cambridge University Press, Cambridge, 61.

[50] Fred, A. (2002) Origins of Existence: How Life Emerged in the Universe. The Free Press, New York, 102.

[51] LeBlanc, F. (2010) An Introduction to Stellar Astrophysics. John Wiley \& Sons, Hoboken, 218.

[52] Lewis, J.S. (2004) Physics and Chemistry of the Solar System. Elsevier Academic 
Press, Amsterdam, 600.

[53] "Deuterium Burning in Substellar Objects". IOPscience. The American Astronomical Society. Retrieved 2 January 2015.

[54] Mollière, P. and Mordasini, C. (2012) Deuterium Burning in Objects Forming via the Core Accretion Scenario. Astronomy \& Astrophysics, 547, A105. https://doi.org/10.1051/0004-6361/201219844

[55] Bodenheimer, P., D’Angelo, G., Lissauer, J.J., Fortney, J.J. and Saumon, D. (2013) Deuterium Burning in Massive Giant Planets and Low-Mass Brown Dwarfs Formed by Core-Nucleated Accretion. The Astrophysical Journal, 770, 120. https://doi.org/10.1088/0004-637X/770/2/120

[56] Rolfs, C.E. and Rodney, W.S. (1988) Cauldrons in the Cosmos: Nuclear Astrophysics. University of Chicago Press, Chicago, 338.

[57] Bethe, H.A. (1939) Energy Production in Stars. Physical Review, 55, 434-456. https://doi.org/10.1103/PhysRev.55.434 